\title{
CONVERGENCE ANALYSIS OF A CLASS OF MASSIVELY PARALLEL DIRECTION SPLITTING ALGORITHMS FOR THE NAVIER-STOKES EQUATIONS IN SIMPLE DOMAINS
}

\author{
JEAN-LUC GUERMOND, PETER D. MINEV, AND ABNER J. SALGADO \\ Abstract. We provide a convergence analysis for a new fractional time- \\ stepping technique for the incompressible Navier-Stokes equations based on \\ direction splitting. This new technique is of linear complexity, unconditionally \\ stable and convergent, and suitable for massive parallelization.
}

\section{INTRODUCTION}

This work is concerned with the analysis of a new class of approximation techniques for the solution of the time-dependent incompressible Navier-Stokes equations based on direction splitting. The complexity of this new technique is linear since it requires to solve a sequence of one-dimensional problems at each time step. The main claims of this paper are that this technique is unconditionally stable and superlinearly convergent with respect to the time discretization parameter and is suitable for massive parallelization.

We consider the Stokes equations written in terms of velocity $\mathfrak{u}$ and pressure $\mathfrak{p}$ on a finite time interval $[0, T]$ and in a cubic domain $\Omega=(0,1)^{d}$ with $d=2$ or 3 :

$$
\begin{cases}\mathfrak{u}_{t}-\Delta \mathfrak{u}+\nabla \mathfrak{p}=f, & \text { in } \Omega \times(0, T], \\ \nabla \cdot \mathfrak{u}=0, & \text { in } \Omega \times[0, T], \\ \left.\mathfrak{u}\right|_{\partial \Omega}=0, & \text { in }(0, T], \\ \left.\mathfrak{u}\right|_{t=0}=\mathfrak{u}_{0}, & \text { in } \Omega,\end{cases}
$$

where $f$ is a smooth source term and $\mathfrak{u}_{0}$ is a solenoidal initial velocity field with zero normal trace. The nonlinear term in the momentum equation of the Navier-Stokes equations is not accounted for since it does not interfere with the incompressibility constraint. The fluid density is assumed to be constant and has been put into the normalization constants.

Received by the editor January 16, 2011 and, in revised form, June 16, 2011.

2010 Mathematics Subject Classification. Primary 65N12, 65N15, 35Q30.

Key words and phrases. Navier-Stokes, fractional time-stepping, direction splitting.

This material is based upon work supported by the National Science Foundation grants DMS0713829, by the Air Force Office of Scientific Research, USAF, under grant/contract number FA9550-09-1-0424, and a Discovery grant of the National Science and Engineering Research Council of Canada. This publication is also partially based on work supported by Award No. KUS-C1-016-04, made by King Abdullah University of Science and Technology (KAUST).

The work of P. Minev was also supported by fellowships from the Institute of Applied Mathematics and Computational Science and the Institute of Scientific Computing at Texas A\&M University.

The work of A.J. Salgado was also been supported by NSF grants CBET-0754983 and DMS0807811 . 
Once time is discretized, (1.1) reduces to a generalized Stokes system at each time step. Solving this coupled system often proves computer intensive and is not easy to solve efficiently in parallel due to the saddle point structure induced by the incompressibility constraint. Alternative more efficient approaches consist of uncoupling the velocity and the pressure using so-called projection algorithms.

Projection algorithms date back to the late 1960s and stem from the seminal works of Chorin [4] and Temam [27. These methods and various improvements thereof are still, to the best of our knowledge, the methods of choice in the CFD community. Although in the 1980s and 1990s these techniques underwent some evolution and their properties are now fairly well understood [19, 24, 25, 26, 29, 12, 9] (the reader is referred to [10] for an overview), the same fundamental idea of decomposing vector fields into a divergence-free part and a gradient has remained unchanged over the years and has been challenged only recently in [13. For all these schemes, the total cost per time step is that of solving one vector-valued advection-diffusion equation and one scalar-valued Poisson equation with homogeneous Neumann boundary conditions. For very large size problems, the cost of solving the Poisson equation is dominant. To address this issue, Guermond and Minev have proposed a new method in [11. The main idea consists of abandoning the projection paradigm, as in [13, and replacing the Poisson equation by a direction splitting strategy. This requires to solve a sequence of one-dimensional elliptic problems instead of one multidimensional Poisson equation. The first-order accurate variant of method has been shown to be unconditionally stable in [11.

In this paper we pursue further the ideas introduced/announced in [11] in the sense that in addition to splitting the pressure-correction, we also apply a direction splitting technique to the momentum equation, thus further reducing the overall computational cost of the method. We prove that the totally split method is convergent and we provide error estimates.

Applying direction splitting to the momentum equation is not a new idea. For instance, in [28, Section 3.7.2] Temam studies a projection method where the solution of the momentum equation is obtained using direction splitting and the incompressibility constraint is enforced by means of a Poisson equation. Stability and convergence of the scheme are proved therein but no error estimates are provided. Lu, Neittaanmäki and Tai show in [20, 21] that this scheme is $\mathcal{O}\left(\tau^{\frac{1}{2}}\right)$ accurate, $\tau$ being the time-step. Our work differs from these previous results mainly in two directions. First, we adopt a direction splitting strategy for the computation of the pressure-correction which renders the method extremely fast and massively parallelizable. Second, we provide error estimates for the proposed scheme, and we show that the so-called standard version of the scheme is $\mathcal{O}(\tau)$-accurate in all quantities irrespective of the space dimension and the rotational version is $\mathcal{O}\left(\tau^{\frac{3}{2}}\right)$-accurate in two space dimensions. Numerical experiments show that the result holds true also in three space dimensions and the actual convergence rate is higher than $\mathcal{O}\left(\tau^{\frac{3}{2}}\right)$ in two and three space dimensions. The algorithm has been implemented in a parallel code which has been observed to have optimal weak scalability. This code has been used to compute the transient regime on the three-dimensional lid-driven cavity at $R_{e}=1000$ and $R_{e}=5000$ on a mesh composed of $2 \times 10^{9}$ nodes on 512 processors only.

This paper is organized as follows. Section 1.1 introduces the notation and establishes some preliminary results. The new algorithm is described in Section 2 , 
two-dimensional and three-dimensional variants of the algorithm are presented in $\$ 2.1$ and $\$ 2.2$, respectively. The convergence analysis of the standard form of the algorithm is done in Section 3 and the analysis of the rotational form is done in Section 4. In Section 5 we briefly discuss the BDF2 technique to march in time. Finally, we present numerical experiments in Section 6 to illustrate the performance of this new class of algorithms.

1.1. Notation and preliminaries. We consider the time-dependent Stokes system (1.1) on the finite time interval $[0, T]$ and in the cubic domain $\Omega:=(0,1)^{d}$ with $d=2$ or 3 .

We henceforth consider only the time discretization of the system to simplify the discussion. Handling the space discretization is a secondary issue, and the reader is referred to [9, 14] for the techniques that can be used for this purpose. Let $\tau>0$ be a time step (for simplicity taken uniform) and let $t_{k}=k \tau$ for $0 \leq k \leq K:=\lceil T / \tau\rceil$. Let $E$ be a normed space, with norm $\|\cdot\|_{E}$. For any time-dependent function $\psi:[0, T] \rightarrow E$, we denote $\psi^{k}:=\psi\left(t_{k}\right)$ and the sequence $\left\{\psi^{k}\right\}_{k=0, \ldots, K}$ is denoted by $\psi_{\tau}$. To simplify the notation we define the time-increment operator $\delta$ acting on time sequences by setting

$$
\delta \psi^{k}:=\psi^{k}-\psi^{k-1},
$$

and we define the time-average operation as follows:

$$
\bar{\psi}^{k+\frac{1}{2}}:=\frac{\psi^{k+1}+\psi^{k}}{2} .
$$

We also define the following discrete norms:

$$
\left\|\psi_{\tau}\right\|_{\ell^{2}(E)}:=\left(\tau \sum_{k=0}^{K}\left\|\psi^{k}\right\|_{E}^{2}\right)^{\frac{1}{2}}, \quad\left\|\psi_{\tau}\right\|_{\ell^{\infty}(E)}:=\max _{0 \leq k \leq K}\left\{\left\|\psi^{k}\right\|_{E}\right\} .
$$

The space of functions $\psi:[0, T] \longrightarrow E$ that are such that the map $(0, T) \ni t \longrightarrow$ $\|\psi(t)\|_{E} \in \mathbb{R}$ is $L^{p}$-integrable is indifferently denoted $L^{p}((0, T) ; E)$ or $L^{p}(E)$.

No notational distinction is made between scalar or vector-valued functions but spaces of vector-valued functions are identified with bold fonts. We use the standard Sobolev spaces $W^{m, p}(\Omega)$, for $0 \leq m \leq \infty$ and $1 \leq p \leq \infty$. The closure with respect to the norm $\|\cdot\|_{W^{m, p}}$ of the space of $\mathcal{C}^{\infty}$-functions compactly supported in $\Omega$ is denoted $W_{0}^{m, p}(\Omega)$. To simplify the notation, the Hilbert space $W^{s, 2}(\Omega)$ (resp. $\left.W_{0}^{s, 2}(\Omega)\right)$ is denoted $H^{s}(\Omega)\left(\operatorname{resp} . H_{0}^{s}(\Omega)\right)$. We define $L_{f=0}^{2}(\Omega)\left(\operatorname{resp} . H_{f=0}^{1}(\Omega)\right)$ the space that is composed of those functions in $L^{2}(\Omega)$ (resp. $H^{1}(\Omega)$ ) that are of zero mean. The scalar product of $\mathbf{L}^{2}(\Omega)$ and $L_{f=0}^{2}(\Omega)$ is denoted $\langle\cdot, \cdot\rangle$ and we define

$$
\begin{aligned}
&\|q\|_{H^{1}}:=\|\nabla q\|_{\mathbf{L}^{2}}, \quad \forall q \in H_{f=0}^{1}(\Omega), \\
&\|v\|_{\mathbf{H}^{1}}:=\|\nabla v\|_{\mathbf{L}^{2}}, \quad \forall v \in \mathbf{H}_{0}^{1}(\Omega) .
\end{aligned}
$$

Finally, we recall that

$$
\|v\|_{\mathbf{H}^{1}}^{2}=\|\nabla \cdot v\|_{L^{2}}^{2}+\|\nabla \times v\|_{\mathbf{L}^{2}}^{2}, \quad \forall v \in \mathbf{H}_{0}^{1}(\Omega) .
$$

Henceforth $c$ denotes a generic constant whose value may change at each occurrence. This constant may depend on the data of the problem and its exact solution, but it does not depend on the discretization parameter $\tau$ or the solution of the numerical scheme. 
1.2. The pressure operator. We assume that we have at hand an unbounded closed operator $A: D(A) \subset L_{f=0}^{2}(\Omega) \rightarrow L_{f=0}^{2}(\Omega)$ with domain $D(A) \subset H_{f=0}^{1}(\Omega)$ and we assume that the following holds:

$$
\begin{cases}\|\nabla q\|_{\mathbf{L}^{2}}^{2} \leq\langle A q, q\rangle, & \forall q \in D(A), \\ \langle A p, q\rangle=\langle p, A q\rangle, & \forall p, q \in(A) .\end{cases}
$$

This property implies that the map $D(A) \ni q \mapsto\|q\|_{A} \in \mathbb{R}$ where

$$
\|q\|_{A}:=\langle A q, q\rangle^{\frac{1}{2}}, \quad \forall q \in D(A),
$$

is a norm. We also define the scalar product

$$
\langle p, q\rangle_{A}:=\langle A p, q\rangle, \quad \forall p, q \in D(A) .
$$

Finally, we introduce the Hilbert space $Y$ to be the completion of the space of smooth scalar-valued functions with respect to the norm $\|\cdot\|_{A}$ :

$$
Y:=\overline{\mathcal{C}} \boldsymbol{\infty}(\Omega)^{\|\cdot\|_{A}} \cap L_{f=0}^{2}(\Omega) .
$$

The extension of the scalar product $\langle\cdot, \cdot\rangle_{A}$ to $Y$ is abusively denoted $\langle\cdot, \cdot\rangle_{A}$.

The symmetry and coercivity hypotheses (1.8) for the operator $A$ are the only hypotheses required for the stability and convergence results stated in this paper to hold true unless explicitly mentioned otherwise.

1.2.1. Example 1: A natural choice for $A$ consists of setting $A=-\Delta_{N}$, where $\Delta_{N}$ is the Laplace operator supplemented with homogeneous Neumann boundary conditions. This operator is the workhorse of classical projection methods. The quasi-totality of the literature dedicated to projections methods uses this operator. Another less known legitimate choice is $A=I-\Delta_{N}$ where $I$ is the identity operator.

1.2.2. Example 2: The main originality of the method that we are going to consider is that incomplete factorizations of $I-\Delta_{N}$ are legitimate also. In two space dimensions we define

(1.12)

$\left\{\begin{array}{l}A:=\left(1-\partial_{x x}\right)\left(1-\partial_{y y}\right), \\ D(A):=\left\{p \in H_{f=0}^{1}(\Omega): \partial_{y y} p, A p \in L^{2}(\Omega):\left.\partial_{y} p\right|_{y=0,1}=0,\left.\partial_{x}\left(1-\partial_{y y}\right) p\right|_{x=0,1}=0\right\},\end{array}\right.$

and in three dimensions

$(1.13)$

$$
\left\{\begin{array}{l}
A:=\left(1-\partial_{x x}\right)\left(1-\partial_{y y}\right)\left(1-\partial_{z z}\right), \\
D(A):=\left\{p \in H_{f=0}^{1}(\Omega): \partial_{z z} p,\left(1-\partial_{y y}\right)\left(1-\partial_{z z}\right) p, A p \in L^{2}(\Omega):\right. \\
\left.\left.\quad \partial_{z} p\right|_{z=0,1}=0,\left.\partial_{y}\left(1-\partial_{z z}\right) p\right|_{y=0,1}=0,\left.\partial_{x}\left(1-\partial_{y y}\right)\left(1-\partial_{z z}\right) p\right|_{x=0,1}=0\right\},
\end{array}\right.
$$

The graph norm is denoted $\|\cdot\|_{D(A)}$ both in two and three space dimensions.

Proposition 1.1. The operator A defined in (1.12) or (1.13), in two or three space dimensions, respectively, satisfies (1.8).

Proof. See [1].

One interesting feature of the operators defined by (1.12) and (1.13) is that solving the equation $A p=g$ for $g \in L^{2}(\Omega)$ only requires to solve one-dimensional 
problems. For instance, the solution of $A p=g$ in three space dimensions is obtained by solving for $p_{1}, p_{2}$, and $p$ so that

$$
\begin{aligned}
p_{1}-\partial_{x x} p_{1} & =g, & \left.\partial_{x} p_{1}\right|_{x=0,1} & =0, \\
p_{2}-\partial_{y y} p_{2} & =p_{1}, & \left.\partial_{y} p_{2}\right|_{y=0,1} & =0, \\
p-\partial_{z z} p & =p_{2}, & \left.\partial_{z} p\right|_{z=0,1} & =0 .
\end{aligned}
$$

When $A$ is defined as in (1.12) or (1.13), the space $Y$ is characterized as follows:

$$
Y= \begin{cases}\left\{q \in H_{f=0}^{1}(\Omega) ; \partial_{x y} q \in L^{2}(\Omega)\right\}, & \text { in } \mathbb{R}^{2}, \\ \left\{q \in H_{f=0}^{1}(\Omega) ; \partial_{x y} q, \partial_{y z} q, \partial_{z x} q, \partial_{x y z} q \in L^{2}(\Omega)\right\} & \text { in } \mathbb{R}^{3} .\end{cases}
$$

Note that the boundary conditions associated with $D(A)$ have disappeared from $Y$ and the $\|\cdot\|_{A}$-norm (which is also the norm in $Y$ ) is characterized by

$$
\|q\|_{A}^{2}= \begin{cases}\|q\|_{H^{1}}^{2}+\left\|\partial_{x y} q\right\|_{L^{2}}^{2}, & \text { in } \mathbb{R}^{2}, \\ \|q\|_{H^{1}}^{2}+\left\|\partial_{x y} q\right\|_{L^{2}}^{2}+\left\|\partial_{y z} q\right\|_{L^{2}}^{2}+\left\|\partial_{z x} q\right\|_{L^{2}}^{2}+\left\|\partial_{x y z} q\right\|_{L^{2}}^{2}, & \text { in } \mathbb{R}^{3} .\end{cases}
$$

1.2.3. Example 3: As a final example, let $\mathcal{A}$ be either the operator $\Delta_{N}$ or $I-\Delta_{N}$ (or suitable discretizations thereof), and let $\Pi$ be a symmetric preconditioner of $\mathcal{A}$ that we assume to be uniformly spectrally equivalent to $\mathcal{A}$. In other words, let us assume that there are constants $c_{1}$ and $c_{2}$ independent of the mesh size so that the following inequalities hold:

$$
c_{1}\left\langle\Pi^{-1} q, q\right\rangle \leq\langle\mathcal{A} q, q\rangle \leq c_{2}\left\langle\Pi^{-1} q, q\right\rangle, \quad \forall q \in D\left(\Pi^{-1}\right) \cap D(\mathcal{A}) .
$$

Then, it is clear that for all $\gamma \geq \frac{1}{c_{2}}$ (i.e., $\gamma$ large enough) the operator $A:=\gamma \Pi^{-1}$ satisfies the symmetry and coercivity hypotheses (1.8). Note that the solution of the equation $A p=g$ reduces to $p=\gamma^{-1} \Pi g$ which is just an application of the preconditioner, which, by design, is easy to perform.

Natural choices for $\Pi$ are the so-called BPX-type preconditioners (see [2] and 3 , Chapter II, Section 4] for the proof of uniform spectral equivalence) or the multigrid $\mathcal{V}$-cycle with a variable number of smoothing steps per level (see [3, Chapter II, Section 7.4]).

1.3. Direction splitting for the velocity. To be able to handle the two-dimensional and three-dimensional error analysis in a unified framework we introduce the following unbounded closed operator

$$
B v:= \begin{cases}\partial_{x x y y} v & \text { in } \mathbb{R}^{2}, \\ \left(\partial_{x x y y}+\partial_{y y z z}+\partial_{z z x x}-\frac{\tau}{2} \partial_{x x y y z z}\right) v & \text { in } \mathbb{R}^{3},\end{cases}
$$

with domain

$$
D(B):=\left\{v \in \mathbf{H}_{0}^{1}(\Omega) ; B v \in \mathbf{L}^{2}(\Omega)\right\} .
$$

The graph norm is denoted $\|\cdot\|_{D(B)}$.

Lemma 1.1. The bilinear form $D(B) \times D(B) \ni(v, w) \longmapsto\langle v, B w\rangle \in \mathbb{R}$ is symmetric positive and the following holds for all $v \in D(B)$ :

$$
\langle v, B v\rangle= \begin{cases}\left\|\partial_{x y} v\right\|_{\mathbf{L}^{2}}^{2} & \text { in } \mathbb{R}^{2}, \\ \left\|\partial_{x y} v\right\|_{\mathbf{L}^{2}}^{2}+\left\|\partial_{y z} v\right\|_{\mathbf{L}^{2}}^{2}+\left\|\partial_{x z} v\right\|_{\mathbf{L}^{2}}^{2}+\frac{\tau}{2}\left\|\partial_{x y z} v\right\|_{\mathbf{L}^{2}}^{2} & \text { in } \mathbb{R}^{3} .\end{cases}
$$


Proof. Let us consider the two-dimensional case first. Using the Fubini-Tonelli Theorem and integrating by parts repeatedly we obtain

$$
\begin{aligned}
\left\langle\partial_{x x y y} v, v\right\rangle & =\int_{x=0}^{x=1}\left[\left.v \partial_{y x x} v\right|_{y=0} ^{y=1}-\int_{y=0}^{y=1} \partial_{y} v \partial_{y x x} v \operatorname{diff} y\right] \operatorname{diff} x \\
& =-\int_{y=0}^{y=1}\left[\left.\partial_{y} v \partial_{y x} v\right|_{x=0} ^{x=1}-\int_{x=0}^{x=1}\left(\partial_{y x} v\right)^{2} \operatorname{diff} x\right] \operatorname{diff} y \\
& =\left\|\partial_{x y} v\right\|_{\mathbf{L}^{2}}^{2} .
\end{aligned}
$$

Note that we used $\left.v\right|_{y=0,1}=0$ and $\left.\partial_{y} v\right|_{x=0,1}=0$ which is a consequence of $\left.v\right|_{x=0,1}=$ 0 . The three-dimensional result is obtained similarly; the details are left to the reader.

To simplify notation we now define the norm

$$
\|v\|_{B}:=\langle v, B v\rangle^{\frac{1}{2}}, \quad v \in D(B),
$$

and we define the Hilbert space

$$
\mathbf{Z}:=\overline{\mathcal{C}^{\infty}(\Omega)} \|^{\|\cdot\|_{B}} \cap \mathbf{H}_{0}^{1}(\Omega) .
$$

The extension of the scalar product $\langle\cdot, \cdot\rangle_{B}$ to $\mathbf{Z}$ is abusively denoted $\langle\cdot, \cdot\rangle_{B}$.

1.4. The right-inverse of the Stokes operator. To describe solenoidal vector fields we introduce the classical spaces

$$
\mathbf{H}:=\left\{v \in \mathbf{L}^{2}(\Omega): \nabla \cdot v=0,\left.v \cdot n\right|_{\partial \Omega}=0\right\}, \quad \mathbf{V}:=\mathbf{H} \cap \mathbf{H}_{0}^{1}(\Omega),
$$

where $n$ is the outer unit normal to $\partial \Omega$ and we denote by $P_{\mathbf{H}}$ the $\mathbf{L}^{2}$-projection onto $\mathbf{H}$. It is also useful to introduce the right-inverse of the Stokes operator $S$ : $\mathbf{L}^{2}(\Omega) \rightarrow \mathbf{V}$ defined as follows: for any $f \in \mathbf{L}^{2}(\Omega)$ we denote $(S f, q) \in \mathbf{V} \times L_{f=0}^{2}(\Omega)$ the pair such that

$$
\begin{cases}-\Delta S f+\nabla q=f, & \text { in } \Omega, \\ \nabla \cdot S f=0, & \text { in } \Omega, \\ S f=0, & \text { on } \partial \Omega .\end{cases}
$$

Given the particular domain that we consider in this work, the inverse Stokes operator is bounded from $\mathbf{L}^{2}(\Omega)$ to $\mathbf{H}^{2} \cap \mathbf{V}$ (cf. [5]), i.e., $\|S f\|_{\mathbf{H}^{2}} \leq c\|f\|_{\mathbf{L}^{2}}$. Finally, we introduce the semi-norm

$$
|v|_{\star}^{2}:=\langle S v, v\rangle, \quad \forall v \in \mathbf{L}^{2}(\Omega),
$$

and, we recall (see e.g. [26, 9, 15]), that for every $\gamma \in(0,1)$, there exists $c(\gamma) \geq 0$ so that the following holds for every $v \in \mathbf{H}_{0}^{1}(\Omega)$ :

$$
\langle\nabla S v, \nabla v\rangle \geq(1-\gamma)\|v\|_{\mathbf{L}^{2}}^{2}-c(\gamma)\left\|v-P_{\mathbf{H}} v\right\|_{\mathbf{L}^{2}}^{2} .
$$

\section{Description of the SCHEMe}

We describe the direction splitting algorithm in two and three space dimensions in this section. The stability and convergence analysis is done in the subsequent sections. 
2.1. Two space dimensions. To simplify the presentation, we assume for the time being that the space dimension is two $(d=2)$ and we defer to $\$ 2.2$ the discussion of the three-dimensional case.

The scheme computes three sequences of variables $\left\{u^{k}\right\},\left\{\phi^{k-\frac{1}{2}}\right\}$, and $\left\{p^{k-\frac{1}{2}}\right\}$ that approximate the velocity, the pressure-correction, and the pressure, respectively.

- Pressure predictor: Denoting by $\mathfrak{p}_{0}$ the exact pressure field at $t=0$ and $\phi^{\star,-\frac{1}{2}}$ an approximation of $\frac{1}{2} \tau \partial_{t} \mathfrak{p}(0)$, the algorithm is initialized by setting $p^{-\frac{1}{2}}=\mathfrak{p}_{0}$ and $\phi^{-\frac{1}{2}}=\phi^{\star,-\frac{1}{2}}$. Then, for all $k \geq 0$ a pressure predictor is computed as follows:

$$
p^{\star, k+\frac{1}{2}}=p^{k-\frac{1}{2}}+\phi^{k-\frac{1}{2}} .
$$

- Velocity update: The velocity field is initialized by setting $u^{0}=\mathfrak{u}_{0}$, and for all $k \geq 0$ the velocity update is computed by solving the following series of one-dimensional problems: Find $\xi^{k+1}, \eta^{k+1}$, and $u^{k+1}$ such that

$$
\begin{aligned}
& \frac{\xi^{k+1}-u^{k}}{\tau}-\Delta u^{k}+\nabla p^{\star, k+\frac{1}{2}}=f^{k+\frac{1}{2}},\left.\quad \xi^{k+1}\right|_{\partial \Omega}=0, \\
& \frac{\eta^{k+1}-\xi^{k+1}}{\tau}-\frac{1}{2} \partial_{x x}\left(\eta^{k+1}-u^{k}\right)=0,\left.\quad \eta^{k+1}\right|_{x=0,1}=0, \\
& \frac{u^{k+1}-\eta^{k+1}}{\tau}-\frac{1}{2} \partial_{y y}\left(u^{k+1}-u^{k}\right)=0,\left.\quad u^{k+1}\right|_{y=0,1}=0 .
\end{aligned}
$$

- Penalty step: The pressure-correction $\phi^{k+\frac{1}{2}}$ is computed by solving

$$
A \phi^{k+\frac{1}{2}}=-\frac{1}{\tau} \nabla \cdot u^{k+1} .
$$

- Pressure update: The last sub-step of the algorithm consists of updating the pressure as follows:

$$
p^{k+\frac{1}{2}}=p^{k-\frac{1}{2}}+\phi^{k+\frac{1}{2}}-\chi \nabla \cdot \bar{u}^{k+\frac{1}{2}} .
$$

Remark 2.1. Note that we use two different sets of symbols to distinguish the exact solution of (1.1), say $(\mathfrak{u}, \mathfrak{p})$, from the approximate solution $(u, p)$.

Remark 2.2. The parameter $\chi \geq 0$ in (2.6) is user dependent. By analogy with the projection-based pressure correction schemes, we say that the method is in standard form if $\chi=0$ and the method is in rotational form if $\chi>0$.

Remark 2.3. By making the linear combinations $(2.2)+(2.3)$ and $(2.2)+(2.3)+$ $2 \times(2.4)$ and by setting $u^{k+\frac{1}{2}}:=\frac{1}{2}\left(\eta^{k+1}+u^{k}\right)$, we observe that the splitting of the momentum equation in (2.2)-(2.3)-(2.4) is equivalent to original alternating directions (ADI) scheme of Peaceman and Rachford, see [22]:

$$
\begin{gathered}
\frac{u^{k+\frac{1}{2}}-u^{k}}{\tau / 2}-\partial_{x x} u^{k+\frac{1}{2}}-\partial_{y y} u^{k}+\nabla p^{\star, k+\frac{1}{2}}=f^{k+\frac{1}{2}},\left.\quad u^{k+\frac{1}{2}}\right|_{x=0,1}=0, \\
\frac{u^{k+1}-u^{k+\frac{1}{2}}}{\tau / 2}-\partial_{x x} u^{k+\frac{1}{2}}-\partial_{y y} u^{k+1}+\nabla p^{\star, k+\frac{1}{2}}=f^{k+\frac{1}{2}},\left.\quad u^{k+1}\right|_{y=0,1}=0 .
\end{gathered}
$$

Remark 2.4. The quantity $\phi^{\star,-\frac{1}{2}}$ can be estimated in many ways. For instance, one can take $\phi^{\star,-\frac{1}{2}}=0$; this limits the convergence of the scheme to first-order. One can also take $\phi^{\star,-\frac{1}{2}}=\mathfrak{p}^{\star, \frac{1}{2}}-\mathfrak{p}_{0}$ where is $\mathfrak{p}^{\star, \frac{1}{2}}$ is an estimate of $\mathfrak{p}\left(\frac{\tau}{2}\right)$. 
Remark 2.5. During the review of this paper it has come to our attention that somewhat similar lower-order versions of the above algorithm have been explored in the Russian literature, e.g. see algorithm (13.6.12)-(13.6.13) from [30].

A remarkable feature of the algorithm (2.1) to (2.6) is that, although the Dirichlet boundary condition on the velocity is not enforced on the entire boundary at the integer time steps, it is indeed fulfilled as claimed in the following.

Proposition 2.1. Let $\left\{u^{k}\right\}$ be the velocity sequence from the algorithm (2.1) to (2.6). Then $\left.u^{k}\right|_{\partial \Omega}=0$ for all $k=0, \ldots, K$.

Proof. Let us prove this by induction. The boundary condition is satisfied by $u^{0}$ by construction. It is clear also by definition of $u^{k+1}$ that the boundary condition is satisfied at $y=0,1$. Let us consider (2.4) at $x=1$, the other boundary can be treated similarly. The boundary condition at $x=1$ on the quantity $\eta^{k+1}$ implies that

$$
u^{k+1}(1, y)=\frac{\tau}{2} \partial_{y y}\left(u^{k+1}(1, y)-u^{k}(1, y)\right)
$$

Moreover, the boundary conditions on $u^{k+1}$ and $u^{k}$ at $y=0$ and $y=1$ imply that the above equation can be rewritten into the following form:

$$
u^{k+1}(1, y)-u^{k}(1, y)-\frac{\tau}{2} \partial_{y y}\left(u^{k+1}-u^{k}\right)(1, y)=0,\left.\quad\left(u^{k+1}-u^{k}\right)(1, \cdot)\right|_{y=0,1}=0
$$

This immediately implies that $u^{k+1}(1, y)=u^{k}(1, y)=0$, which concludes the proof.

This result turns out to be crucial for the error analysis.

2.2. Three space dimensions. The purpose of this section is to propose a threedimensional version of the above splitting technique. Since the alternating directions method of Peaceman and Rachford described in [22] (i.e., (2.7)-(2.8) ) does not extend to three dimensions, we use the alternating directions method proposed by Douglas [6] instead to approximate the momentum equation.

The algorithm is again composed of four steps: pressure predictor, velocity update, penalty step, pressure update.

- Pressure predictor: Denoting by $\mathfrak{p}_{0}$ the pressure field at $t=0$ and $\phi^{\star,-\frac{1}{2}}$ an approximation of $\frac{1}{2} \tau \partial_{t} \mathfrak{p}(0)$, the algorithm is initialized by setting $p^{-\frac{1}{2}}=\mathfrak{p}_{0}$ and $\phi^{-\frac{1}{2}}=\phi^{\star,-\frac{1}{2}}$. Then for all $k \geq 0$ a pressure predictor is computed as follows:

$$
p^{\star, k+\frac{1}{2}}=p^{k-\frac{1}{2}}+\phi^{k-\frac{1}{2}}
$$

- Velocity update: The velocity field is initialized by setting $u^{0}=\mathfrak{u}_{0}$, and for all $k \geq 0$ the velocity update is computed by solving the following series of 
one-dimensional problems: Find $\xi^{k+1}, \eta^{k+1}, \zeta^{k+1}$, and $u^{k+1}$ such that

$$
\begin{aligned}
& \frac{\xi^{k+1}-u^{k}}{\tau}-\Delta u^{k}+\nabla p^{\star, k+\frac{1}{2}}=f^{k+\frac{1}{2}},\left.\quad \xi^{k+1}\right|_{\partial \Omega}=0, \\
& \frac{\eta^{k+1}-\xi^{k+1}}{\tau}-\frac{1}{2} \partial_{x x}\left(\eta^{k+1}-u^{k}\right)=0,\left.\quad \eta^{k+1}\right|_{x=0,1}=0, \\
& \frac{\zeta^{k+1}-\eta^{k+1}}{\tau}-\frac{1}{2} \partial_{y y}\left(\zeta^{k+1}-u^{k}\right)=0,\left.\quad \zeta^{k+1}\right|_{y=0,1}=0, \\
& \frac{u^{k+1}-\zeta^{k+1}}{\tau}-\frac{1}{2} \partial_{z z}\left(u^{k+1}-u^{k}\right)=0,\left.\quad u^{k+1}\right|_{z=0,1}=0 .
\end{aligned}
$$

- Penalty step: The pressure-correction $\phi^{k+\frac{1}{2}}$ is computed by solving

$$
A \phi^{k+\frac{1}{2}}=-\frac{1}{\tau} \nabla \cdot u^{k+1} .
$$

- Pressure update: The last sub-step of the algorithm consists of updating the pressure as follows:

$$
p^{k+\frac{1}{2}}=p^{k-\frac{1}{2}}+\phi^{k+\frac{1}{2}}-\chi \nabla \cdot \bar{u}^{k+\frac{1}{2}} .
$$

Remark 2.6. As mentioned in Section 1, the advection term in the full NavierStokes equations does not pose any particular theoretical difficulty with respect to the pressure-velocity splitting, and we do not include it in the numerical analysis to avoid unnecessary technicalities. There are several ways to incorporate the advection into the scheme. The simplest technique consists of treating it explicitly with a second- or third-order Adams-Bashforth scheme; this introduces a CFL-type stability condition, which in our opinion is reasonable and does not spoil the parallel performance of the algorithm (see [16, 17] for details). In all computations presented below (see Section 6), the momentum equation (2.2) in $2 \mathrm{D}$ and (2.10) in $3 \mathrm{D}$ is replaced by

$$
\frac{\xi^{k+1}-u^{k}}{\tau}-\frac{1}{R_{e}} \Delta u^{k}+\nabla p^{*, k+\frac{1}{2}}+\mathbf{N L}^{k+1}\left(u^{k}, u^{k-1}\right)=f^{k+\frac{1}{2}},\left.\quad \xi^{k+1}\right|_{\partial \Omega}=a^{k+1},
$$

where $\mathbf{N L}^{k+1}\left(u^{k}, u^{k-1}\right):=\frac{3}{2}\left(u^{k} \cdot \nabla\right) u^{k}-\frac{1}{2}\left(u^{k-1} \cdot \nabla\right) u^{k-1}$ and $a^{k+1}$ is the Dirichlet boundary condition on the velocity at time level $k+1$. Moreover, the pressure correction is rewritten as follows to properly account for the presence of the Reynolds number:

$$
p^{k+\frac{1}{2}}=p^{k-\frac{1}{2}}+\phi^{k+\frac{1}{2}}-\frac{\chi}{R_{e}} \nabla \cdot \bar{u}^{k+\frac{1}{2}} .
$$

In conclusion the full algorithm is (2.9)-(2.16)-(2.10)-(2.11)-(2.12)-(2.13)-(2.14)(2.17) when solving the nonlinear Navier-Stokes equations in three space dimensions.

Remark 2.7. The above method is an extension of the alternating direction method proposed by Douglas 6]. In order to see this, we add (2.10) and (2.11) to obtain

$$
\frac{\eta^{k+1}-u^{k}}{\tau}-\frac{1}{2} \partial_{x x}\left(\eta^{k+1}+u^{k}\right)-\left(\partial_{y y}+\partial_{z z}\right) u^{k}+\nabla p^{\star, k+\frac{1}{2}}=f^{k+\frac{1}{2}}
$$

Adding (2.10)-(2.12) we obtain

$$
\frac{\zeta^{k+1}-u^{k}}{\tau}-\frac{1}{2} \partial_{x x}\left(\eta^{k+1}+u^{k}\right)-\frac{1}{2} \partial_{y y}\left(\zeta^{k+1}+u^{k}\right)-\partial_{z z} u^{k}+\nabla p^{\star, k+\frac{1}{2}}=f^{k+\frac{1}{2}}
$$


Finally, adding (2.10) -2.13 we obtain

$\frac{u^{k+1}-u^{k}}{\tau}-\frac{1}{2} \partial_{x x}\left(\eta^{k+1}+u^{k}\right)-\frac{1}{2} \partial_{y y}\left(\zeta^{k+1}+u^{k}\right)-\frac{1}{2} \partial_{z z}\left(u^{k+1}+u^{k}\right)+\nabla p^{\star, k+\frac{1}{2}}=f^{k+\frac{1}{2}}$.

These equations correspond to (3.1a)-(3.1c) of [6], respectively.

Proposition 2.2. Let $\left\{u^{k}\right\}$ be the velocity sequence from the algorithm (2.9)(2.15). Then $\left.u^{k}\right|_{\partial \Omega}=0$ for all $k=0, \ldots, K$.

$$
\left.u^{k}\right|_{\partial \Omega}=0, \quad \forall k \geq 0 .
$$

Proof. Proceed as in the proof of Proposition 2.1

2.3. Compatibility conditions. Note that $\mathfrak{p}_{0}:=\left.\mathfrak{p}\right|_{t=0}$ is not part of the initial data but this quantity can be computed by solving

$$
\Delta \mathfrak{p}_{0}=\nabla \cdot\left(f_{0}+\Delta \mathfrak{u}_{0}\right),\left.\quad \partial_{n} \mathfrak{p}_{0}\right|_{\Gamma}=\left(f_{0}+\Delta \mathfrak{u}_{0}\right) \cdot n,
$$

where we have set $f_{0}:=\left.f\right|_{t=0}$. This then requires the initial data to satisfy the following compatibility condition at the boundary $\left.\left(-\Delta \mathfrak{u}_{0}+\nabla \mathfrak{p}_{0}-f_{0}\right)\right|_{\Gamma}=0$ which we assume to hold. This condition holds, for instance, if $\mathfrak{u}_{0}=0$ and $f_{0}=0$, i.e., the fluid is at rest at $t=0$ and the source term is zero at $t=0$. If the above compatibility condition is not satisfied, the error analysis must be adapted to account for weighted error estimates by proceeding as in [18, 23].

\section{ERROR ANALYSIS OF THE STANDARD SCHEME}

The purpose of this section is to study the convergence of the algorithms (2.7)(2.6) in two space dimensions and (2.9) - (2.15) in three space dimensions for $\chi=$ 0 . The main claim of this section is that the standard version of our scheme is unconditionally stable and first-order convergent in all quantities.

3.1. Consistency of the momentum equation. To evaluate the consistency error on the momentum equation, we rewrite the momentum equation in a more recognizable Crank-Nicolson form. This is done in two space dimensions by adding (2.2), (2.3) and (2.4) as follows:

$$
\frac{u^{k+1}-u^{k}}{\tau}-\frac{1}{2} \partial_{y y} u^{k+1}-\frac{1}{2} \partial_{x x} \eta^{k+1}-\frac{1}{2} \Delta u^{k}+\nabla p^{\star, k+\frac{1}{2}}=f^{k+\frac{1}{2}} .
$$

Then using (2.4) to eliminate $\eta^{k+1}$ from the above equality, i.e., $\eta^{k+1}=u^{k+1}-$ $\frac{\tau}{2} \partial_{y y}\left(u^{k+1}-u^{k}\right)$, we obtain the following evolution equation for the integer steps,

$$
\frac{u^{k+1}-u^{k}}{\tau}-\Delta \bar{u}^{k+\frac{1}{2}}+\nabla p^{\star, k+\frac{1}{2}}+\frac{\tau}{4} \partial_{x x y y} \delta u^{k+1}=f^{k+\frac{1}{2}}
$$

where the time increment $\delta(\cdot)$ and the time averaging $\overline{(\cdot)}$ operators are defined in (1.2) and (1.3), respectively.

The same trick can be used in three space dimensions as suggested in [6]. By proceeding as above, the intermediate steps, $\xi^{k+1}, \eta^{k+1}$, and $\zeta^{k+1}$, can be eliminated, so that the momentum equation becomes:

$$
\begin{aligned}
\frac{u^{k+1}-u^{k}}{\tau}-\Delta \bar{u}^{k+\frac{1}{2}} & +\frac{\tau}{4}\left(\partial_{x x y y}+\partial_{y y z z}+\partial_{z z x x}-\frac{\tau}{2} \partial_{x x y y z z}\right) \delta u^{k+1} \\
& +\nabla p^{\star, k+\frac{1}{2}}=f^{k+\frac{1}{2}}
\end{aligned}
$$


Owing to the definition of the operator $B$ (see (1.17), the momentum equation can be rewritten as follows independently of the space dimension:

$$
\frac{u^{k+1}-u^{k}}{\tau}-\Delta \bar{u}^{k+\frac{1}{2}}+\frac{\tau}{4} B \delta u^{k+1}+\nabla p^{\star, k+\frac{1}{2}}=f^{k+\frac{1}{2}} .
$$

3.2. Consistency analysis of the algorithm. Let $\mathfrak{u}, \mathfrak{p}$ be the solution of (1.1). We define the following velocity and pressure errors:

$$
e^{k+1}:=\mathfrak{u}^{k+1}-u^{k+1}, \quad \epsilon^{k+\frac{1}{2}}:=\mathfrak{p}^{k+\frac{1}{2}}-p^{k+\frac{1}{2}},
$$

where $\mathfrak{u}^{k+1}:=\mathfrak{u}\left(t_{k+1}\right)$ and $\mathfrak{p}^{k+\frac{1}{2}}:=\mathfrak{p}\left(t_{k+\frac{1}{2}}\right)$.

Next, we obtain equations controlling the errors. Since $\chi=0$, the pressure update implies that the pressure predictor can also be written as follows:

$$
p^{\star, k+\frac{1}{2}}=2 p^{k-\frac{1}{2}}-p^{k-\frac{3}{2}}
$$

that is, the pressure predictor is a second-order extrapolation of the pressure at time level $k+\frac{1}{2}$. Upon subtracting (3.4) from the momentum equation (1.1), we obtain

$$
\left(1+\frac{\tau^{2}}{4} B\right)\left(e^{k+1}-e^{k}\right)-\tau \Delta \bar{e}^{k+\frac{1}{2}}+\tau \nabla \epsilon^{\star, k+\frac{1}{2}}=\tau \mathcal{R}^{k+\frac{1}{2}},
$$

where we have set $\epsilon^{\star, k+\frac{1}{2}}:=2 \epsilon^{k-\frac{1}{2}}-\epsilon^{k-\frac{3}{2}}$ and the residual $\mathcal{R}^{k+\frac{1}{2}}$ is defined by

$$
\mathcal{R}^{k+\frac{1}{2}}=\left[\frac{\delta \mathfrak{u}^{k+1}}{\tau}-\left(\mathfrak{u}_{t}\right)^{k+\frac{1}{2}}\right]-\Delta\left[\overline{\mathfrak{u}}^{k+\frac{1}{2}}-\mathfrak{u}^{k+\frac{1}{2}}\right]-\nabla\left[\mathfrak{p}^{k+\frac{1}{2}}-\mathfrak{p}^{\star, k+\frac{1}{2}}\right]+\frac{\tau}{4} B\left[\delta \mathfrak{u}^{k+1}\right],
$$

where we have again set $\mathfrak{p}^{\star, k+\frac{1}{2}}:=2 \mathfrak{p}^{k-\frac{1}{2}}-\mathfrak{p}^{k-\frac{3}{2}}$. Finally, using (2.6) (or (2.15)) with $\chi=0$ to eliminate $\phi^{k+\frac{1}{2}}$ from (2.5) (or (2.14)) and using the incompressibility constraint, we obtain

$$
\left\langle\delta \epsilon^{k+\frac{1}{2}}, q\right\rangle_{A}=\frac{1}{\tau}\left\langle e^{k+1}, \nabla q\right\rangle+\left\langle\delta \mathfrak{p}^{k+\frac{1}{2}}, q\right\rangle_{A}, \quad \forall q \in Y .
$$

Note that it is not legitimate to write the equality in strong form, i.e., $A \delta \epsilon^{k+\frac{1}{2}}$ is equal to $-\frac{1}{\tau} \nabla \cdot e^{k+1}+A \delta \mathfrak{p}^{k+\frac{1}{2}}$ since $\delta \mathfrak{p}^{k+\frac{1}{2}}$ is not in $D(A)$ (i.e., $\delta \mathfrak{p}^{k+\frac{1}{2}}$ does not satisfy the artificial boundary conditions associated with $D(A)$ ).

Lemma 3.1. Let $\mathfrak{u} \in W^{2, \infty}\left(\mathbf{H}^{2}(\Omega)\right) \cap W^{1, \infty}(D(B))$ and $\mathfrak{p} \in W^{2, \infty}\left(H^{1}(\Omega)\right)$. Then

$$
2 \tau\left\langle\mathcal{R}^{k+\frac{1}{2}}, v\right\rangle \leq c \tau^{5}+\frac{\tau}{4}\|v\|_{\mathbf{L}^{2}}^{2}, \quad \forall v \in \mathbf{L}^{2}(\Omega) .
$$

Proof. Each of the terms in $\mathcal{R}^{k+\frac{1}{2}}$ is $\mathcal{O}\left(\tau^{2}\right)$, given the smoothness of the exact solution. Note that $\mathfrak{p}^{k+\frac{1}{2}}-\mathfrak{p}^{\star, k+\frac{1}{2}}=\delta^{2} \mathfrak{p}^{k+\frac{1}{2}}$.

3.3. First-order estimates on the velocity. Let us assume that the quantity $\phi^{\star, \frac{1}{2}}$ is estimated so that the following holds:

$$
\left\|\mathfrak{p}\left(\frac{\tau}{2}\right)-p^{\star}, \frac{1}{2}\right\|_{L^{2}} \leq c \tau .
$$

This is the case if $\phi^{\star, \frac{1}{2}}=0$ and if the pressure is smooth enough, say $\mathfrak{p} \in$ $\mathcal{C}^{0}\left([0, T], L^{2}(\Omega)\right)$. Then, the main result of this section is the following first-order convergence statement. 
Theorem 3.1. Assume that the solution $(\mathfrak{u}, \mathfrak{p})$ to (1.1) is smooth enough $($ say $\mathfrak{u} \in$ $W^{2, \infty}\left(\mathbf{H}^{2}(\Omega)\right) \cap W^{1, \infty}(D(B))$ and $\left.\mathfrak{p} \in W^{2, \infty}(Y)\right)$. Then, provided that (3.11) holds, the solution $\left(u_{\tau}, p_{\tau}\right)$ to the discrete scheme (2.1) -(2.6) in two space dimensions and (2.9) - 2.15) in three space dimensions, with $\chi=0$, satisfies the following error estimate:

$$
\left\|e_{\tau}\right\|_{\ell^{\infty}\left(\mathbf{L}^{2}\right)}+\left\|e_{\tau}\right\|_{\ell^{2}\left(\mathbf{H}^{1}\right)}+\tau\left\|e_{\tau}\right\|_{\ell^{\infty}(B)}+\tau\left\|\epsilon_{\tau}\right\|_{\ell^{\infty}(A)}+\sqrt{\tau}\left\|\delta e_{\tau}\right\|_{\ell^{2}(B)} \leq c \tau .
$$

Proof. Multiply equation (3.7) by $2 e^{k+1}$ and integrate over $\Omega$. Since both the exact velocity and the approximate one at integer time steps satisfy the full boundary conditions, we obtain

$$
\begin{gathered}
\left(1-\frac{\tau}{4}\right)\left\|e^{k+1}\right\|_{\mathbf{L}^{2}}^{2}+\left\|\delta e^{k+1}\right\|_{\mathbf{L}^{2}}^{2}+\frac{\tau}{2}\left\|e^{k+1}\right\|_{\mathbf{H}^{1}}^{2}+2 \tau\left\|\bar{e}^{k+\frac{1}{2}}\right\|_{\mathbf{H}^{1}}^{2}+2 \tau\left\langle\nabla \epsilon^{\star, k+\frac{1}{2}}, e^{k+1}\right\rangle \\
+\frac{\tau^{2}}{4}\left\|e^{k+1}\right\|_{B}^{2}+\frac{\tau^{2}}{4}\left\|\delta e^{k+1}\right\|_{B}^{2} \leq\left\|e^{k}\right\|_{\mathbf{L}^{2}}^{2}+\frac{\tau}{2}\left\|e^{k}\right\|_{\mathbf{H}^{1}}^{2}+\frac{\tau^{2}}{4}\left\|e^{k}\right\|_{B}^{2}+c \tau^{5},
\end{gathered}
$$

where we have used the identity $2 a(a \pm b)=a^{2}-b^{2}+(a \pm b)^{2}$.

By using $2 \tau^{2} \epsilon^{\star, k+\frac{1}{2}}$ as a test function in (3.9), we obtain

$$
2 \tau^{2}\left\langle\delta \epsilon^{k+\frac{1}{2}}, \epsilon^{\star, k+\frac{1}{2}}\right\rangle_{A}=2 \tau\left\langle e^{k+1}, \nabla \epsilon^{\star, k+\frac{1}{2}}\right\rangle+2 \tau^{2}\left\langle\delta \mathfrak{p}^{k+\frac{1}{2}}, \epsilon^{\star, k+\frac{1}{2}}\right\rangle_{A} .
$$

Clearly,

$$
\left\langle\delta \epsilon^{k+\frac{1}{2}}, \epsilon^{\star, k+\frac{1}{2}}\right\rangle_{A}=\left\langle\delta \epsilon^{k+\frac{1}{2}}, \epsilon^{k+\frac{1}{2}}\right\rangle_{A}-\left\langle\delta \epsilon^{k+\frac{1}{2}}, \delta^{2} \epsilon^{k+\frac{1}{2}}\right\rangle_{A},
$$

so that using again the identity $2 a(a-b)=a^{2}-b^{2}+(a-b)^{2}$ we obtain

$$
\begin{aligned}
\tau^{2}\left[\left\|\epsilon^{k+\frac{1}{2}}\right\|_{A}^{2}-\left\|\epsilon^{k-\frac{1}{2}}\right\|_{A}^{2}+\left\|\delta \epsilon^{k-\frac{1}{2}}\right\|_{A}^{2}\right]-\tau^{2}\left\|\delta^{2} \epsilon^{k+\frac{1}{2}}\right\|_{A}^{2} \\
=2 \tau\left\langle e^{k+1}, \nabla \epsilon^{\star, k+\frac{1}{2}}\right\rangle+2 \tau^{2}\left\langle\delta \mathfrak{p}^{k+\frac{1}{2}}, \epsilon^{\star, k+\frac{1}{2}}\right\rangle_{A}
\end{aligned}
$$

To obtain a control on $\left\|\delta^{2} \epsilon^{k+\frac{1}{2}}\right\|_{A}^{2}$, we apply the time increment operator $\delta$ to (3.9) (assuming that $k \geq 2$ ) and we use the test function $\tau \delta^{2} \epsilon^{k+\frac{1}{2}}$ :

$$
\begin{aligned}
& \tau\left\|\delta^{2} \epsilon^{k+\frac{1}{2}}\right\|_{A}^{2}=\left\langle\delta e^{k+1}, \nabla \delta^{2} \epsilon^{k+\frac{1}{2}}\right\rangle+\tau\left\langle\delta^{2} \mathfrak{p}^{k+\frac{1}{2}}, \delta^{2} \epsilon^{k+\frac{1}{2}}\right\rangle_{A} \\
& \leq\left\|\delta e^{k+1}\right\|_{\mathbf{L}^{2}}\left\|\nabla \delta^{2} \epsilon^{k+\frac{1}{2}}\right\|_{\mathbf{L}^{2}}+\tau\left\|\delta^{2} \mathfrak{p}^{k+\frac{1}{2}}\right\|_{A}\left\|\delta^{2} \epsilon^{k+\frac{1}{2}}\right\|_{A} \\
& \leq\left(\left\|\delta e^{k+1}\right\|_{\mathbf{L}^{2}}+\tau\left\|\delta^{2} \mathfrak{p}^{k+\frac{1}{2}}\right\|_{A}\right)\left\|\delta^{2} \epsilon^{k+\frac{1}{2}}\right\|_{A},
\end{aligned}
$$

so that

$$
\tau^{2}\left\|\delta^{2} \epsilon^{k+\frac{1}{2}}\right\|_{A}^{2} \leq\left\|\delta e^{k+1}\right\|_{\mathbf{L}^{2}}^{2}+\tau^{2}\left\|\delta^{2} \mathfrak{p}^{k+\frac{1}{2}}\right\|_{A}^{2}+2 \tau\left\|\delta^{2} \mathfrak{p}^{k+\frac{1}{2}}\right\|_{A}\left\|\delta e^{k+1}\right\|_{\mathbf{L}^{2}} .
$$

Adding (3.13), (3.14) and (3.15) we obtain

$$
\begin{aligned}
(1 & \left.-\frac{\tau}{4}\right)\left\|e^{k+1}\right\|_{\mathbf{L}^{2}}^{2}+\frac{\tau}{2}\left\|e^{k+1}\right\|_{\mathbf{H}^{1}}^{2}+2 \tau\left\|\bar{e}^{k+\frac{1}{2}}\right\|_{\mathbf{H}^{1}}^{2}+\tau^{2}\left[\left\|\epsilon^{k+\frac{1}{2}}\right\|_{A}^{2}+\left\|\delta \epsilon^{k-\frac{1}{2}}\right\|_{A}^{2}\right] \\
& +\frac{\tau^{2}}{4}\left[\left\|e^{k+1}\right\|_{B}^{2}+\left\|\delta e^{k+1}\right\|_{B}^{2}\right] \leq c \tau^{5}+\left\|e^{k}\right\|_{\mathbf{L}^{2}}^{2}+\frac{\tau}{2}\left\|e^{k}\right\|_{\mathbf{H}^{1}}^{2}+\tau^{2}\left\|\epsilon^{k-\frac{1}{2}}\right\|_{A}^{2} \\
& +\frac{\tau^{2}}{4}\left\|e^{k}\right\|_{B}^{2}+2 \tau^{2}\left\langle\delta \mathfrak{p}^{k+\frac{1}{2}}, \epsilon^{\star, k+\frac{1}{2}}\right\rangle_{A}+\tau^{2}\left\|\delta^{2} \mathfrak{p}^{k+\frac{1}{2}}\right\|_{A}^{2}+2 \tau\left\|\delta^{2} \mathfrak{p}^{k+\frac{1}{2}}\right\|_{A}\left\|\delta e^{k+1}\right\|_{\mathbf{L}^{2}}
\end{aligned}
$$


Let us examine the last three terms in detail:

- $\tau^{2}\left\|\delta^{2} \mathfrak{p}^{k+\frac{1}{2}}\right\|_{A}^{2}$. Given the smoothness of $\mathfrak{p}$ this term is $\mathcal{O}\left(\tau^{5}\right)$.

- $2 \tau\left\|\delta^{2} \mathfrak{p}^{k+\frac{1}{2}}\right\|_{A}\left\|\delta e^{k+1}\right\|_{\mathbf{L}^{2}}$. We estimate it as follows:

$2 \tau\left\|\delta^{2} \mathfrak{p}^{k+\frac{1}{2}}\right\|_{A}\left\|\delta e^{k+1}\right\|_{\mathbf{L}^{2}} \leq c \tau^{3}\left(\left\|e^{k+1}\right\|_{\mathbf{L}^{2}}+\left\|e^{k}\right\|_{\mathbf{L}^{2}}\right) \leq c \tau^{5}+\frac{\tau}{4}\left\|e^{k+1}\right\|_{\mathbf{L}^{2}}^{2}+\frac{\tau}{2}\left\|e^{k}\right\|_{\mathbf{L}^{2}}^{2}$.

- $2 \tau^{2}\left\langle A \delta \mathfrak{p}^{k+\frac{1}{2}}, \epsilon^{\star, k+\frac{1}{2}}\right\rangle$. Given the smoothness of $\mathfrak{p}$ we obtain

$$
\begin{aligned}
2 \tau^{2}\left\langle\delta \mathfrak{p}^{k+\frac{1}{2}}, \epsilon^{\star, k+\frac{1}{2}}\right\rangle_{A} & =2 \tau^{2}\left\langle\delta \mathfrak{p}^{k+\frac{1}{2}}, \epsilon^{k-\frac{1}{2}}\right\rangle_{A}+2 \tau^{2}\left\langle\delta \mathfrak{p}^{k+\frac{1}{2}}, \delta \epsilon^{k-\frac{1}{2}}\right\rangle_{A} \\
& \leq c \tau^{3}\left\|\epsilon^{k-\frac{1}{2}}\right\|_{A}+c \tau^{3}\left\|\delta \epsilon^{k-\frac{1}{2}}\right\|_{A} \\
& \leq c \tau^{3}+\tau^{3}\left\|\epsilon^{k-\frac{1}{2}}\right\|_{A}^{2}+\tau^{2}\left\|\delta \epsilon^{k-\frac{1}{2}}\right\|_{A}^{2} .
\end{aligned}
$$

Note that this term is the only one in the entire error analysis that spoils the game. This consistency term does not allow us to obtain directly an error estimate of order larger than $\mathcal{O}(\tau)$.

We have finally proved that the following holds for all $k \geq 2$ :

$$
\begin{aligned}
&\left(1-\frac{\tau}{2}\right)\left\|e^{k+1}\right\|_{\mathbf{L}^{2}}^{2}+\frac{\tau}{2}\left\|e^{k+1}\right\|_{\mathbf{H}^{1}}^{2}+2 \tau\left\|\bar{e}^{k+\frac{1}{2}}\right\|_{\mathbf{H}^{1}}^{2}+\tau^{2}\left\|\epsilon^{k+\frac{1}{2}}\right\|_{A}^{2} \\
&+\frac{\tau^{2}}{4}\left[\left\|e^{k+1}\right\|_{B}^{2}+\left\|\delta e^{k+1}\right\|_{B}^{2}\right] \leq c \tau^{3}+\left(1+\frac{\tau}{2}\right)\left\|e^{k}\right\|_{\mathbf{L}^{2}}^{2}+\frac{\tau}{2}\left\|e^{k}\right\|_{\mathbf{H}^{1}}^{2} \\
&+\tau^{2}(1+\tau)\left\|\epsilon^{k-\frac{1}{2}}\right\|_{A}^{2}+\frac{\tau^{2}}{4}\left\|e^{k}\right\|_{B}^{2} .
\end{aligned}
$$

Upon observing that the initialization process $\left(p^{-\frac{1}{2}}=\mathfrak{p}_{0}\right)$ implies

$$
\tau^{2}\left\|\delta^{2} \epsilon^{\frac{3}{2}}\right\|_{A}^{2} \leq(1+\tau)\left\|e^{2}\right\|^{2}+\tau^{3},
$$

we infer that the above inequality holds also for $k=1$. As a consequence of (3.11), we also deduce that

$$
\left\|e^{1}\right\|_{\mathbf{L}^{2}}^{2}+\tau\left\|e^{1}\right\|_{\mathbf{H}^{1}}^{2}+\tau\left\|e^{\frac{1}{2}}\right\|_{\mathbf{H}^{1}}^{2}+\tau^{2}\left\|\epsilon^{\frac{1}{2}}\right\|_{A}^{2}+\tau^{2}\left\|e^{1}\right\|_{B}^{2} \leq c \tau^{4} .
$$

By summing the above relation from $k=1$ to $K$ and by applying the discrete Grönwall lemma allows us to conclude.

The ability of $\delta u^{k+1} / \tau$ to approximate $\mathfrak{u}_{t}$ is made explicit in the following.

Lemma 3.2. Let the solution $(\mathfrak{u}, \mathfrak{p})$ to (1.1) be smooth enough such that $\mathfrak{u} \in W^{3, \infty}\left(\mathbf{H}^{2}(\Omega)\right) \cap W^{2, \infty}(D(B))$ and $\mathfrak{p} \in W^{3, \infty}(Y)$. Then the solution $\left(u_{\tau}, p_{\tau}\right)$ to the discrete scheme (2.1)-(2.6) in two space dimensions and (2.9)-(2.15) in three space dimensions, with $\chi=0$, satisfies the following error estimate:

$$
\left\|\delta e_{\tau}\right\|_{\ell^{\infty}\left(\mathbf{L}^{2}\right)}+\left\|\delta e_{\tau}\right\|_{\ell^{2}\left(\mathbf{H}^{1}\right)}+\tau\left\|\delta \epsilon_{\tau}\right\|_{\ell^{\infty}(A)}+\tau\left\|\delta e_{\tau}\right\|_{\ell^{\infty}(B)} \leq c \tau^{2} .
$$

Proof. Apply the arguments in the proof of Theorem 3.1 to the time increments.

3.4. Error estimates on the pressure. It is known that for the incremental projection scheme in standard form it is possible to prove that the error on the pressure in the $\ell^{2}\left(L^{2}\right)$-norm is $\mathcal{O}(\tau)$ (cf. [9, 10, 26]). The purpose of this paragraph is to show that, although on a weaker norm, a similar result holds for the proposed algorithm. Let us define the norm

$$
\|q\|_{\triangle}=\sup _{0 \neq v \in \mathbf{Z}} \frac{\langle\nabla q, v\rangle}{\|v\|_{\mathbf{Z}}} .
$$


Theorem 3.2. Assume that the hypotheses of Lemma 3.2 hold, then

$$
\left\|\epsilon_{\tau}\right\|_{\ell^{2}(\triangle)} \leq c \tau \text {. }
$$

Proof. Using the error equation (3.7) we obtain

$$
\begin{aligned}
& \left\|\epsilon^{\star, k+\frac{1}{2}}\right\|_{\triangle}=\sup _{0 \neq v \in \mathbf{Z}} \frac{1}{\|v\|_{\mathbf{Z}}}\left[\left\langle\frac{\delta e^{k+1}}{\tau}, v\right\rangle+\left\langle\nabla \bar{e}^{k+\frac{1}{2}}, \nabla v\right\rangle+\frac{\tau}{4}\left\langle\delta e^{k+1}, v\right\rangle_{B}+\left\langle\mathcal{R}^{k+\frac{1}{2}}, v\right\rangle\right] \\
& \leq \frac{\left\|\delta e^{k+1}\right\|_{\mathbf{L}^{2}}}{\tau}+\left\|e^{k+\frac{1}{2}}\right\|_{\mathbf{H}^{1}}+\frac{\tau}{4}\left\|\delta e^{k+1}\right\|_{B}+c \tau^{2} \\
& \leq c \tau+\left\|\bar{e}^{k+\frac{1}{2}}\right\|_{\mathbf{H}^{1}},
\end{aligned}
$$

where the last estimate holds in view of Lemma 3.2. Take the square of this inequality, multiply it by $\tau$ and sum over $k$. The result follows by using the conclusion of Theorem 3.1 .

Remark 3.1. It seems that it may be possible to obtain a first-order error estimate on the pressure in the $\ell^{2}\left(L^{2}\right)$-norm in the fully discrete case under the additional (somewhat restrictive) condition

$$
\tau \leq \begin{cases}c h & \text { in } \mathbb{R}^{2}, \\ c h^{\frac{4}{3}} & \text { in } \mathbb{R}^{3} .\end{cases}
$$

This is a CFL condition in two space dimensions. The reasoning behind this conjecture is the following. Assume that the velocity is approximated using a finitedimensional space $\mathbf{X}_{h}$ and that the norm in $B$ is appropriately approximated, say $\|\cdot\|_{B_{h}}$. In view of (1.19) it is reasonable to expect that the following inverse inequalities hold:

$$
\|v\|_{B_{h}} \leq\left\{\begin{array}{ll}
c h^{-1}\|v\|_{\mathbf{H}^{1}} & \text { in } \mathbb{R}^{2}, \\
c h^{-1}\left(1+\tau^{\frac{1}{2}} h^{-1}\right)\|v\|_{\mathbf{H}^{1}} & \text { in } \mathbb{R}^{3},
\end{array} \quad \forall v \in \mathbf{X}_{h} .\right.
$$

Then, assuming that the pressure is approximated using a space $M_{h} \subset H_{f=0}^{1}(\Omega)$ so that the pair $\left(\mathbf{X}_{h}, M_{h}\right)$ satisfies the so-called LBB condition, [7, 8, we obtain

$$
\begin{aligned}
& c\left\|\epsilon^{\star, k+\frac{1}{2}}\right\|_{L^{2}} \leq \sup _{\substack{0 \neq v \in \mathbf{X}_{h}\\
}} \frac{\left\langle\nabla \epsilon^{\star, k+\frac{1}{2}}, v\right\rangle}{\|v\|_{\mathbf{H}^{1}}} \\
& \leq \sup _{0 \neq v \in \mathbf{X}_{h}} \frac{1}{\|v\|_{\mathbf{H}^{1}}}\left[\left\langle\delta e^{k+1} / \tau, v\right\rangle+\left\langle\nabla \bar{e}^{k+\frac{1}{2}}, \nabla v\right\rangle+\frac{\tau}{4}\left\langle\delta e^{k+1}, v\right\rangle_{B_{h}}+\left\langle\mathcal{R}^{k+\frac{1}{2}}, v\right\rangle\right] \\
& \leq\left\|\delta e^{k+1} / \tau\right\|_{\mathbf{L}^{2}}+\left\|\bar{e}^{k+\frac{1}{2}}\right\|_{\mathbf{H}^{1}}+\frac{\tau}{4} \sup _{0 \neq v \in \mathbf{X}_{h}} \frac{\left\|\delta e^{k+1}\right\|_{B_{h}}\|v\|_{B_{h}}}{\|v\|_{\mathbf{H}^{1}}}+c \tau^{2} .
\end{aligned}
$$

The two-dimensional inverse inequality implies

$$
\left\|\epsilon^{\star, k+\frac{1}{2}}\right\|_{L^{2}} \leq c \tau^{2}+\left\|\tau^{-1} \delta e^{k+1}\right\|_{\mathbf{L}^{2}}+\left\|\bar{e}^{k+\frac{1}{2}}\right\|_{\mathbf{H}^{1}}+c \tau^{2} h^{-2}\left\|\tau^{-1} \delta e^{k+1}\right\|_{\mathbf{H}^{1}},
$$

whereas the three-dimensional inverse inequality implies

$$
\left\|\epsilon^{\star, k+\frac{1}{2}}\right\|_{L^{2}} \leq c \tau^{2}+\left\|\tau^{-1} \delta e^{k+1}\right\|_{\mathbf{L}^{2}}+\left\|\bar{e}^{k+\frac{1}{2}}\right\|_{\mathbf{H}^{1}}+c\left(\tau^{2} h^{-2}+\tau^{3} h^{-4}\right)\left\|\tau^{-1} \delta e^{k+1}\right\|_{\mathbf{H}^{1}} .
$$

Take the square of this inequality, multiply it by $\tau$ and sum over $k$, then the estimates of Lemma 3.2 together with condition (3.19) yield the desired estimate, $\left\|\epsilon_{\tau}\right\|_{\ell^{2}\left(L^{2}\right)} \leq c \tau$. 
3.5. Second-order estimates on the velocity. Despite the fact that numerical experiments suggest that the standard form of the above algorithm is close to second-order on the velocity in the $\mathbf{L}^{2}$-norm, (see Section 6), a proof of such a statement eludes us at the moment. We briefly elaborate in this section on the difficulties that arise when trying to establish a second-order error estimate.

The argument one usually invokes to prove a second-order error estimate consists of multiplying the error equation by $S \bar{e}^{k+\frac{1}{2}}$, where $S$ is the right-inverse Stokes operator (see (1.23) ). Following this reasoning, and using property (1.25), we obtain that the following holds

$$
\begin{aligned}
\frac{1}{2}\left(\left|e^{k+1}\right|_{\star}^{2}-\left|e^{k}\right|_{\star}^{2}\right)+\frac{3 \tau}{4}\left\|\bar{e}^{k+\frac{1}{2}}\right\|_{\mathbf{L}^{2}}^{2}+\frac{\tau^{2}}{4}\left\langle\delta e^{k+1}, S \bar{e}^{k+\frac{1}{2}}\right\rangle_{B} \\
\quad \leq \tau\left\langle\mathcal{R}^{k+\frac{1}{2}}, S \bar{e}^{k+1}\right\rangle+c \tau\left\|\bar{e}^{k+\frac{1}{2}}-P_{\mathbf{H}} \bar{e}^{k+\frac{1}{2}}\right\|_{\mathbf{L}^{2}}^{2} .
\end{aligned}
$$

Provided the exact solution is smooth enough, we can estimate the residual term in a way similar to Lemma 3.1 .

$$
\tau\left\langle\mathcal{R}^{k+\frac{1}{2}}, S \bar{e}^{k+\frac{1}{2}}\right\rangle \leq c \tau^{5}+\frac{\tau}{8}\left\|\bar{e}^{k+\frac{1}{2}}\right\|_{\mathbf{L}^{2}}^{2} .
$$

Using the estimates of Lemma 3.2 we can control the $B$-norm as follows:

$$
\left|\frac{\tau^{2}}{4}\left\langle\delta e^{k+1}, S \bar{e}^{k+\frac{1}{2}}\right\rangle_{B}\right| \leq \frac{\tau^{2}}{4}\left\|\delta e^{k+1}\right\|_{B}\left\|S \bar{e}^{k+\frac{1}{2}}\right\|_{B} \leq c \tau^{3}\left\|S \bar{e}^{k+\frac{1}{2}}\right\|_{B} .
$$

In two space dimensions the $\mathbf{H}^{2}$-regularity of $S$ implies $\left\|S \bar{e}^{k+\frac{1}{2}}\right\|_{B} \leq c\left\|\bar{e}^{k+\frac{1}{2}}\right\|_{\mathbf{L}^{2}}$ so that

$$
\left|\frac{\tau^{2}}{4}\left\langle\delta e^{k+1}, S \bar{e}^{k+\frac{1}{2}}\right\rangle_{B}\right| \leq c \tau^{5}+\frac{\tau}{8}\left\|\bar{e}^{k+\frac{1}{2}}\right\|_{\mathbf{L}^{2}}^{2} .
$$

Note that the above reasoning does not apply in three space dimensions. In conclusion, in two space dimensions (3.20) becomes

$$
\begin{aligned}
\left|e^{k+1}\right|_{\star}^{2}-\left|e^{k}\right|_{\star}^{2}+\tau\left\|\bar{e}^{k+\frac{1}{2}}\right\|_{\mathbf{L}^{2}}^{2} & \leq c \tau\left(\tau^{4}+\inf _{v \in \mathbf{H}}\left\|\bar{e}^{k+\frac{1}{2}}-v\right\|_{\mathbf{L}^{2}}^{2}\right) \\
& =c \tau\left(\tau^{4}+\left\|\bar{e}^{k+\frac{1}{2}}-P_{\mathbf{H}} \bar{e}^{k+\frac{1}{2}}\right\|_{\mathbf{L}^{2}}^{2}\right),
\end{aligned}
$$

which in turn yields

$$
\left\|\bar{e}_{\tau}\right\|_{\ell^{2}\left(\mathbf{L}^{2}\right)}^{2} \leq c\left(\tau^{4}+\left\|\bar{e}_{\tau}-P_{\mathbf{H}} \bar{e}_{\tau}\right\|_{\ell^{2}\left(\mathbf{L}^{2}\right)}^{2}\right) .
$$

This inequality shows the estimate on $\left\|\bar{e}_{\tau}\right\|_{\ell^{2}\left(\mathbf{L}^{2}\right)}$ is controlled by $\left\|\bar{e}_{\tau}-P_{\mathbf{H}} \bar{e}_{\tau}\right\|_{\ell^{2}\left(\mathbf{L}^{2}\right)}$. Let us now try to bound $\left\|\bar{e}_{\tau}-P_{\mathbf{H}} \bar{e}_{\tau}\right\|_{\ell^{2}\left(\mathbf{L}^{2}\right)}$ uniformly.

By definition, there is $\mu^{k+\frac{1}{2}} \in H_{f=0}^{1}(\Omega)$ so that $\bar{e}^{k+\frac{1}{2}}-P_{\mathbf{H}} \bar{e}^{k+\frac{1}{2}}=\nabla \mu^{k+\frac{1}{2}}$. In other words, $\mu^{k+\frac{1}{2}}$ solves $-\Delta \mu^{k+\frac{1}{2}}=-\nabla \cdot \bar{e}^{k+\frac{1}{2}}$ and $\left.\partial_{n} \mu^{k+\frac{1}{2}}\right|_{\partial \Omega}=0$. Then the penalty equation (3.9) together with the assumed smoothness of the pressure and the estimates of Lemma 3.2 imply that

$$
\begin{aligned}
\| \bar{e}^{k+\frac{1}{2}}- & P_{\mathbf{H}} \bar{e}^{k+\frac{1}{2}}\left\|_{\mathbf{L}^{2}}^{2}=\right\| \nabla \mu^{k+\frac{1}{2}} \|_{\mathbf{L}^{2}}^{2} \\
& =\left\langle\bar{e}^{k+\frac{1}{2}}, \nabla \mu^{k+\frac{1}{2}}\right\rangle=\frac{\tau}{2}\left\langle\delta \epsilon^{k+\frac{1}{2}}-\delta \mathfrak{p}^{k+\frac{1}{2}}+\delta \epsilon^{k-\frac{1}{2}}-\delta \mathfrak{p}^{k-\frac{1}{2}}, \mu^{k+\frac{1}{2}}\right\rangle_{A} \\
& \leq \frac{\tau}{2}\left(\left\|\delta \epsilon^{k+\frac{1}{2}}\right\|_{A}+\left\|\delta \mathfrak{p}^{k+\frac{1}{2}}\right\|_{A}+\left\|\delta \epsilon^{k-\frac{1}{2}}\right\|_{A}+\left\|\delta \mathfrak{p}^{k-\frac{1}{2}}\right\|_{A}\right)\left\|\mu^{k+\frac{1}{2}}\right\|_{A} \\
& \leq c \tau^{2}\left\|\mu^{k+\frac{1}{2}}\right\|_{A} .
\end{aligned}
$$


This finally gives the estimate

$$
\left\|\bar{e}^{k+\frac{1}{2}}-P_{\mathbf{H}} \bar{e}^{k+\frac{1}{2}}\right\|_{\mathbf{L}^{2}} \leq c \tau^{2} \frac{\left\|\mu^{k+\frac{1}{2}}\right\|_{A}}{\left\|\nabla \mu^{k+\frac{1}{2}}\right\|_{\mathbf{L}^{2}}},
$$

which can be controlled uniformly if $\|\cdot\|_{A}$ induces a norm equivalent to $H^{1}$. This is unfortunately not true with the operators $A$ defined in (1.12) and (1.13).

In conclusion, the reasoning carried out above seems to indicate that the rightinverse Stokes operator $S$ is not the correct operator that should be used for the duality argument. The operator that should be used instead still eludes us at the moment.

\section{ERROR ANALYSIS OF THE ROTATIONAL SCHEME}

The purpose of this section is to analyze the algorithms (2.1)-(2.6) and (2.9)(2.15) for $\chi \neq 0$ and to show that, as it is the case for the classical rotational pressure-correction schemes (cf. [15]), these algorithms provide a better order of convergence than the standard form.

4.1. Consistency analysis. Let $\mathfrak{u}, \mathfrak{p}$ be the solution of (1.1). We define the following velocity and pressure errors:

$$
e^{k+1}:=\mathfrak{u}^{k+1}-u^{k+1}, \quad \epsilon^{k+\frac{1}{2}}:=\mathfrak{p}^{k+\frac{1}{2}}-p^{k+\frac{1}{2}},
$$

where $\mathfrak{u}^{k+1}:=\mathfrak{u}\left(t_{k+1}\right)$ and $\mathfrak{p}^{k+\frac{1}{2}}:=\mathfrak{p}\left(t_{k+\frac{1}{2}}\right)$. The error on the pressure correction is measured by introducing the following quantity:

$$
\mathcal{P}^{k+\frac{1}{2}}:=\delta \epsilon^{k+\frac{1}{2}}+\chi \nabla \cdot \bar{e}^{k+\frac{1}{2}} .
$$

Using the above notation we infer

$$
\begin{aligned}
\mathfrak{p}^{k+\frac{1}{2}}-p^{\star, k+\frac{1}{2}} & =\mathfrak{p}^{k+\frac{1}{2}}-\left(p^{k-\frac{1}{2}}+\phi^{k-\frac{1}{2}}\right) \\
& =\mathfrak{p}^{k+\frac{1}{2}}-\left(p^{k-\frac{1}{2}}+p^{k-\frac{1}{2}}-p^{k-\frac{3}{2}}+\chi \nabla \cdot \bar{u}^{k-\frac{1}{2}}\right) \\
& =\delta^{2} \mathfrak{p}^{k+\frac{1}{2}}+\left(\epsilon^{k-\frac{1}{2}}+\delta \epsilon^{k-\frac{1}{2}}+\chi \nabla \cdot \bar{e}^{k-\frac{1}{2}}\right) \\
& =\delta^{2} \mathfrak{p}^{k+\frac{1}{2}}+\epsilon^{k-\frac{1}{2}}+\mathcal{P}^{k-\frac{1}{2}} .
\end{aligned}
$$

Then momentum equation is rewritten as follows:

$$
\left(1+\frac{\tau^{2}}{4} B\right)\left(e^{k+1}-e^{k}\right)-\tau \Delta \bar{e}^{k+\frac{1}{2}}+\tau \nabla\left(\epsilon^{k-\frac{1}{2}}+\mathcal{P}^{k-\frac{1}{2}}\right)=\tau \mathcal{R}^{k+\frac{1}{2}},
$$

where the residual $\mathcal{R}^{k+\frac{1}{2}}$ is defined by

$$
\mathcal{R}^{k+\frac{1}{2}}=\left[\frac{\delta \mathfrak{u}^{k+1}}{\tau}-\left(\mathfrak{u}_{t}\right)^{k+\frac{1}{2}}\right]-\Delta\left[\overline{\mathfrak{u}}^{k+\frac{1}{2}}-\mathfrak{u}^{k+\frac{1}{2}}\right]-\nabla\left[\delta^{2} \mathfrak{p}^{k+\frac{1}{2}}\right]+\frac{\tau}{4} B\left[\delta \mathfrak{u}^{k+1}\right]
$$

The result of Lemma 3.1 holds again, $\mathcal{R}^{k+\frac{1}{2}}=\mathcal{O}\left(\tau^{2}\right)$, provided the exact solution is smooth enough. The equation that controls the pressure correction is rewritten as follows:

$$
\left\langle\mathcal{P}^{k+\frac{1}{2}}, q\right\rangle_{A}=\frac{1}{\tau}\left\langle e^{k+1}, \nabla q\right\rangle+\left\langle\delta \mathfrak{p}^{k+\frac{1}{2}}, q\right\rangle_{A} \quad \forall q \in Y
$$


4.2. A priori estimate on the divergence of the velocity. Let us assume that the quantity $\phi^{\star, \frac{1}{2}}$ is estimated so that the following holds:

$$
\left\|\mathfrak{p}\left(\frac{\tau}{2}\right)-p^{\star}, \frac{1}{2}\right\|_{L^{2}} \leq c \tau .
$$

This is the case if $\phi^{\star, \frac{1}{2}}=0$ and if the pressure is smooth enough, say $\mathfrak{p} \in$ $\mathcal{C}^{0}\left([0, T], L^{2}(\Omega)\right)$. The main result of this section is the following.

Theorem 4.1. Assume that the solution $(\mathfrak{u}, \mathfrak{p})$ to (1.1) is smooth enough, (say $\mathfrak{u} \in W^{2, \infty}\left(\mathbf{H}^{2}(\Omega)\right) \cap W^{1, \infty}(D(B))$ and $\left.\mathfrak{p} \in W^{2, \infty}(Y)\right)$. Then, provided (4.6) holds, the solution $\left(u_{\tau}, p_{\tau}\right)$ to the discrete scheme (2.1) -(2.6) in two space dimensions and (2.9) -(2.15) in three space dimensions, with $0<\chi \leq 1$, satisfies the following error estimate:

$$
\left\|\delta e_{\tau}\right\|_{\ell^{\infty}\left(L^{2}\right)}^{2}+\tau\left\|\nabla \times \delta e_{\tau}\right\|_{\ell^{\infty}\left(\mathbf{L}^{2}\right)}^{2}+\left\|\nabla \times \delta \bar{e}_{\tau}\right\|_{\ell^{2}\left(\mathbf{L}^{2}\right)}^{2}+\tau\left\|\nabla \cdot e_{\tau}\right\|_{\ell^{\infty}\left(L^{2}\right)}^{2} \leq c \tau^{4} .
$$

Proof. Following [15, we derive an improved estimate on the divergence of the velocity. This is done by working with the time increments of (4.3)-(4.5).

Apply the time increment operator $\delta$ to the momentum equation (4.3) and test against $2 \delta e^{k+1}$ to obtain

$$
\begin{aligned}
& \left(1-\frac{\tau}{4}\right)\left\|\delta e^{k+1}\right\|_{\mathbf{L}^{2}}^{2}+\left\|\delta^{2} e^{k+1}\right\|_{\mathbf{L}^{2}}^{2}+\frac{\tau}{2}\left\|\delta e^{k+1}\right\|_{\mathbf{H}^{1}}^{2}+2 \tau\left\|\delta \bar{e}^{k+\frac{1}{2}}\right\|_{\mathbf{H}^{1}}^{2}+\frac{\tau}{4}\left\|\delta e^{k+1}\right\|_{B}^{2} \\
& +2 \tau\left\langle\nabla\left(\delta \epsilon^{k-\frac{1}{2}}+\delta \mathcal{P}^{k-\frac{1}{2}}\right), \delta e^{k+1}\right\rangle \leq c \tau^{5}+\left\|\delta e^{k}\right\|_{\mathbf{L}^{2}}^{2}+\frac{\tau}{2}\left\|\delta e^{k}\right\|_{\mathbf{H}^{1}}^{2}+\frac{\tau}{4}\left\|\delta e^{k}\right\|_{B}^{2}
\end{aligned}
$$

where we used the fact that the residual is $\mathcal{O}\left(\tau^{2}\right)$. Note that we could decrease the consistency error to $\mathcal{O}\left(\tau^{3}\right)$ by assuming more regularity on $\mathfrak{u}$ and $\mathfrak{p}$, but it would not improve the overall accuracy of the method since the splitting error will turn out to be $\mathcal{O}\left(\tau^{2}\right)$ (see below).

Apply the time increment operator $\delta$ to 4.5 and use $2 \tau^{2} \mathcal{P}^{k+\frac{1}{2}}$ as a test function. We obtain

$$
\begin{aligned}
\tau^{2}\left\|\mathcal{P}^{k+\frac{1}{2}}\right\|_{A}^{2}+\tau^{2}\left\|\delta \mathcal{P}^{k+\frac{1}{2}}\right\|_{A}^{2}-\tau^{2}\left\|\mathcal{P}^{k-\frac{1}{2}}\right\|_{A}^{2} \\
\quad=-2 \tau\left\langle\nabla \cdot \delta e^{k+1}, \mathcal{P}^{k+\frac{1}{2}}\right\rangle+2 \tau^{2}\left\langle\delta^{2} \mathfrak{p}^{k+\frac{1}{2}}, \mathcal{P}^{k+\frac{1}{2}}\right\rangle_{A} \\
\quad=-2 \tau\left\langle\nabla \cdot \delta e^{k+1}, \chi \nabla \cdot \bar{e}^{k+\frac{1}{2}}+\delta \epsilon^{k+\frac{1}{2}}\right\rangle+2 \tau^{2}\left\langle\delta^{2} \mathfrak{p}^{k+\frac{1}{2}}, \mathcal{P}^{k+\frac{1}{2}}\right\rangle_{A}
\end{aligned}
$$

where we used the identity $2 a(a \pm b)=a^{2}+(a \pm b)^{2}-b^{2}$. This gives

$$
\begin{aligned}
\tau^{2}\left\|\mathcal{P}^{k+\frac{1}{2}}\right\|_{A}^{2}+ & \tau^{2}\left\|\delta \mathcal{P}^{k+\frac{1}{2}}\right\|_{A}^{2}+\chi \tau\left\|\nabla \cdot e^{k+1}\right\|_{L^{2}}^{2}=\tau^{2}\left\|\mathcal{P}^{k-\frac{1}{2}}\right\|_{A}^{2} \\
& +\chi \tau\left\|\nabla \cdot e^{k}\right\|_{L^{2}}^{2}+2 \tau\left\langle\delta e^{k+1}, \nabla \delta \epsilon^{k+\frac{1}{2}}\right\rangle+2 \tau^{2}\left\langle\delta^{2} \mathfrak{p}^{k+\frac{1}{2}}, \mathcal{P}^{k+\frac{1}{2}}\right\rangle_{A}
\end{aligned}
$$

Apply again the time increment operator $\delta$ to 4.5 and test with $-2 \tau^{2} \delta^{2} \mathcal{P}^{k+\frac{1}{2}}$. Using again, the identity $2 a(a-b)=a^{2}+(a-b)^{2}-b^{2}$, we obtain

$$
\begin{aligned}
-\tau^{2}\left[\left\|\delta \mathcal{P}^{k+\frac{1}{2}}\right\|_{A}^{2}+\left\|\delta^{2} \mathcal{P}^{k+\frac{1}{2}}\right\|_{A}^{2}-\left\|\delta \mathcal{P}^{k-\frac{1}{2}}\right\|_{A}^{2}\right] & =-2 \tau\left\langle\nabla \delta^{2} \mathcal{P}^{k+\frac{1}{2}}, \delta e^{k+1}\right\rangle \\
& -2 \tau^{2}\left\langle\delta^{2} \mathfrak{p}^{k+\frac{1}{2}}, \delta^{2} \mathcal{P}^{k+\frac{1}{2}}\right\rangle_{A} .
\end{aligned}
$$

Observe that (4.9) + (4.10) amounts to testing the time increment of (4.5) with $2 \tau^{2}\left(\mathcal{P}^{k-\frac{1}{2}}+\delta \mathcal{P}^{k-\frac{1}{2}}\right)$. We have split the two steps to make the argument clearer. 
By summing (4.8), (4.9) and (4.10) we deduce that

$$
\begin{aligned}
(1- & \left.\frac{\tau}{4}\right)\left\|\delta e^{k+1}\right\|_{\mathbf{L}^{2}}^{2}+\frac{\tau}{2}\left\|\delta e^{k+1}\right\|_{\mathbf{H}^{1}}^{2}+2 \tau\left\|\delta \bar{e}^{k+\frac{1}{2}}\right\|_{\mathbf{H}^{1}}^{2}+\frac{\tau}{4}\left\|\delta e^{k+1}\right\|_{B}^{2} \\
& +\chi \tau\left\|\nabla \cdot e^{k+1}\right\|_{L^{2}}^{2}+\tau^{2}\left\|\mathcal{P}^{k+\frac{1}{2}}\right\|_{A}^{2}+\tau^{2}\left\|\delta \mathcal{P}^{k-\frac{1}{2}}\right\|_{A}^{2}+\left\|\delta^{2} e^{k+1}\right\|_{\mathbf{L}^{2}}^{2} \\
& -\tau^{2}\left\|\delta^{2} \mathcal{P}^{k+\frac{1}{2}}\right\|_{A}^{2}-2 \chi \tau\left\langle\nabla \cdot \delta \bar{e}^{k+\frac{1}{2}}, \nabla \cdot \delta e^{k+1}\right\rangle \leq c \tau^{5}+\left\|\delta e^{k}\right\|_{\mathbf{L}^{2}}^{2} \\
& +\frac{\tau}{2}\left\|\delta e^{k}\right\|_{\mathbf{H}^{1}}^{2}+\frac{\tau}{4}\left\|\delta e^{k}\right\|_{B}^{2}+\chi \tau\left\|\nabla \cdot e^{k}\right\|_{L^{2}}^{2}+\tau^{2}\left\|\mathcal{P}^{k-\frac{1}{2}}\right\|_{A}^{2} \\
& +2 \tau^{2}\left\langle\delta^{2} \mathfrak{p}^{k+\frac{1}{2}}, \mathcal{P}^{k-\frac{1}{2}}+\delta \mathcal{P}^{k-\frac{1}{2}}\right\rangle_{A},
\end{aligned}
$$

where we used the following identities:

$$
\begin{array}{r}
\epsilon^{k-\frac{1}{2}}+\delta \mathcal{P}^{k-\frac{1}{2}}-\delta \epsilon^{k+\frac{1}{2}}+\delta^{2} \mathcal{P}^{k+\frac{1}{2}}=\chi \nabla \cdot \delta \bar{e}^{k+\frac{1}{2}}, \\
\mathcal{P}^{k+\frac{1}{2}}-\delta^{2} \mathcal{P}^{k+\frac{1}{2}}=\mathcal{P}^{k-\frac{1}{2}}+\delta \mathcal{P}^{k-\frac{1}{2}}
\end{array}
$$

Given the smoothness of $\mathfrak{p}$, the following holds:

$$
2 \tau^{2}\left\langle\delta^{2} \mathfrak{p}^{k+\frac{1}{2}}, \mathcal{P}^{k-\frac{1}{2}}+\delta \mathcal{P}^{k-\frac{1}{2}}\right\rangle_{A} \leq c \tau^{5}+\frac{\tau^{3}}{2}\left\|\mathcal{P}^{k-\frac{1}{2}}\right\|_{A}^{2}+\tau^{2}\left\|\delta \mathcal{P}^{k-\frac{1}{2}}\right\|_{A}^{2} .
$$

Observe that it is here that the irreducible splitting error comes into full light. Although the consistency of the time increment of the momentum equation is $\mathcal{O}\left(\tau^{3}\right)$ (provided enough regularity is assumed on $\mathfrak{u}$ and $\mathfrak{p}$ ), the above inequality shows that the splitting error of the method is $\mathcal{O}\left(\tau^{2}\right)$. Then (4.11) becomes

$$
\begin{aligned}
(1- & \left.\frac{\tau}{4}\right)\left\|\delta e^{k+1}\right\|_{\mathbf{L}^{2}}^{2}+\frac{\tau}{2}\left\|\delta e^{k+1}\right\|_{\mathbf{H}^{1}}^{2}-\chi \frac{\tau}{2}\left\|\nabla \cdot \delta e^{k+1}\right\|_{L^{2}}^{2}+2 \tau\left\|\delta \bar{e}^{k+\frac{1}{2}}\right\|_{\mathbf{H}^{1}}^{2} \\
& -2 \tau \chi\left\|\nabla \cdot \delta \bar{e}^{k+\frac{1}{2}}\right\|_{L^{2}}^{2}+\frac{\tau}{4}\left\|\delta e^{k+1}\right\|_{B}^{2}+\chi \tau\left\|\nabla \cdot e^{k+1}\right\|_{L^{2}}^{2}+\tau^{2}\left\|\mathcal{P}^{k+\frac{1}{2}}\right\|_{A}^{2} \\
& +\left\|\delta^{2} e^{k+1}\right\|_{\mathbf{L}^{2}}^{2}-\tau^{2}\left\|\delta^{2} \mathcal{P}^{k+\frac{1}{2}}\right\|_{A}^{2} \leq c \tau^{5}+\left\|\delta e^{k}\right\|_{\mathbf{L}^{2}}^{2}+\frac{\tau}{2}\left\|\delta e^{k}\right\|_{\mathbf{H}^{1}}^{2} \\
& -\frac{\tau}{2} \chi\left\|\nabla \cdot \delta e^{k}\right\|_{L^{2}}^{2}+\frac{\tau}{4}\left\|\delta e^{k}\right\|_{B}^{2}+\chi \tau\left\|\nabla \cdot e^{k}\right\|_{L^{2}}^{2}+\tau^{2}\left(1+\frac{\tau}{2}\right)\left\|\mathcal{P}^{k-\frac{1}{2}}\right\|_{A}^{2},
\end{aligned}
$$

where we used

$$
-2 \chi\left\langle\nabla \cdot \delta \bar{e}^{k+\frac{1}{2}}, \nabla \cdot \delta e^{k+1}\right\rangle=-\chi\left[\frac{\tau}{2}\left\|\nabla \cdot \delta e^{k+1}\right\|_{L^{2}}^{2}-\frac{\tau}{2}\left\|\nabla \cdot \delta e^{k}\right\|_{L^{2}}^{2}+2 \tau\left\|\nabla \cdot \delta \bar{e}^{k+\frac{1}{2}}\right\|_{L^{2}}^{2}\right] .
$$

Then the identity (1.7) gives

$$
\begin{aligned}
(1- & \left.\frac{\tau}{4}\right)\left\|\delta e^{k+1}\right\|_{\mathbf{L}^{2}}^{2}+\frac{\tau}{2}\left\|\nabla \times \delta e^{k+1}\right\|_{\mathbf{L}^{2}}^{2}+(1-\chi) \frac{\tau}{2}\left\|\nabla \cdot \delta e^{k+1}\right\|_{L^{2}}^{2} \\
& +2 \tau\left\|\nabla \times \delta \bar{e}^{k+\frac{1}{2}}\right\|_{\mathbf{H}^{1}}^{2}+2 \tau(1-\chi)\left\|\nabla \cdot \delta \bar{e}^{k+\frac{1}{2}}\right\|_{L^{2}}^{2}+\frac{\tau}{4}\left\|\delta e^{k+1}\right\|_{B}^{2} \\
& +\chi \tau\left\|\nabla \cdot e^{k+1}\right\|_{L^{2}}^{2}+\tau^{2}\left\|\mathcal{P}^{k+\frac{1}{2}}\right\|_{A}^{2}+\left\|\delta^{2} e^{k+1}\right\|_{\mathbf{L}^{2}}^{2}-\tau^{2}\left\|\delta^{2} \mathcal{P}^{k+\frac{1}{2}}\right\|_{A}^{2} \\
& \leq c \tau^{5}+\left\|\delta e^{k}\right\|_{\mathbf{L}^{2}}^{2}+\frac{\tau}{2}\left\|\nabla \times \delta e^{k}\right\|_{\mathbf{L}^{2}}^{2}+(1-\chi) \frac{\tau}{2}\left\|\nabla \cdot \delta e^{k}\right\|_{L^{2}}^{2}+\frac{\tau}{4}\left\|\delta e^{k}\right\|_{B}^{2} \\
& +\chi \tau\left\|\nabla \cdot e^{k}\right\|_{L^{2}}^{2}+\tau^{2}\left(1+\frac{\tau}{2}\right)\left\|\mathcal{P}^{k-\frac{1}{2}}\right\|_{A}^{2} .
\end{aligned}
$$

To conclude we are going to observe that the quantity $\left\|\delta^{2} e^{k+1}\right\|_{\mathbf{L}^{2}}^{2}-\tau^{2}\left\|\delta^{2} \mathcal{P}^{k+\frac{1}{2}}\right\|_{A}^{2}$ is nonnegative up to some consistency error. To see this, let us apply the time increment operator $\delta^{2}$ to (4.5) and test the equation with $\tau \delta^{2} \mathcal{P}^{k+\frac{1}{2}}$. After using the Cauchy-Schwarz inequality and the inequality (1.8), we obtain

$$
\tau\left\|\delta^{2} \mathcal{P}^{k+\frac{1}{2}}\right\|_{A} \leq\left\|\delta^{2} e^{k+1}\right\|_{\mathbf{L}^{2}}+\tau\left\|\delta^{3} \mathfrak{p}^{k+\frac{1}{2}}\right\|_{A},
$$


which, given the smoothness assumption on $\mathfrak{p}$, then implies

$$
\begin{aligned}
\tau^{2}\left\|\delta^{2} \mathcal{P}^{k+\frac{1}{2}}\right\|_{A}^{2} & \leq\left\|\delta^{2} e^{k+1}\right\|_{\mathbf{L}^{2}}^{2}+\tau^{2}\left\|\delta^{3} \mathfrak{p}^{k+\frac{1}{2}}\right\|_{A}^{2}+2 \tau\left\|\delta^{2} e^{k+1}\right\|_{\mathbf{L}^{2}}\left\|\delta^{3} \mathfrak{p}^{k+\frac{1}{2}}\right\|_{A}, \\
& \leq c \tau^{5}+\left\|\delta^{2} e^{k+1}\right\|_{\mathbf{L}^{2}}^{2}+\frac{\tau}{4}\left\|\delta e^{k+1}\right\|_{\mathbf{L}^{2}}^{2}+\frac{\tau}{2}\left\|\delta e^{k}\right\|_{\mathbf{L}^{2}}^{2}
\end{aligned}
$$

Note again that the consistency error could be decreased to $\mathcal{O}\left(\tau^{3}\right)$ by assuming $\mathfrak{p} \in W^{3, \infty}(Y)$, but this would be useless since the splitting error of the method has been shown to be $\mathcal{O}\left(\tau^{2}\right)$ above. By adding this last inequality to (4.13) we finally obtain that the following holds for all $k \geq 2$ :

$$
\begin{aligned}
(1- & \left.\frac{\tau}{2}\right)\left\|\delta e^{k+1}\right\|_{\mathbf{L}^{2}}^{2}+\frac{\tau}{2}\left\|\nabla \times \delta e^{k+1}\right\|_{\mathbf{L}^{2}}^{2}+(1-\chi) \frac{\tau}{2}\left\|\nabla \cdot \delta e^{k+1}\right\|_{L^{2}}^{2}+2 \tau \| \nabla \\
\times & \delta \bar{e}^{k+\frac{1}{2}}\left\|_{\mathbf{L}^{2}}^{2}+2 \tau(1-\chi)\right\| \nabla \cdot \delta \bar{e}^{k+\frac{1}{2}}\left\|_{L^{2}}^{2}+\frac{\tau}{4}\right\| \delta e^{k+1}\left\|_{B}^{2}+\chi \tau\right\| \nabla \cdot e^{k+1} \|_{L^{2}}^{2} \\
& +\tau^{2}\left\|\mathcal{P}^{k+\frac{1}{2}}\right\|_{A}^{2} \leq c \tau^{5}+\left(1+\frac{\tau}{2}\right)\left\|\delta e^{k}\right\|_{\mathbf{L}^{2}}^{2}+\frac{\tau}{2}\left\|\nabla \times \delta e^{k}\right\|_{\mathbf{L}^{2}}^{2} \\
& +(1-\chi) \frac{\tau}{2}\left\|\nabla \cdot \delta e^{k}\right\|_{L^{2}}^{2}+\frac{\tau}{4}\left\|\delta e^{k}\right\|_{B}^{2}+\chi \tau\left\|\nabla \cdot e^{k}\right\|_{L^{2}}^{2}+\tau^{2}\left(1+\frac{\tau}{2}\right)\left\|\mathcal{P}^{k-\frac{1}{2}}\right\|_{A}^{2} .
\end{aligned}
$$

The following estimate also holds as a consequence of the initialization hypothesis (4.6):

$$
\left\|\delta e^{2}\right\|_{\mathbf{L}^{2}}^{2}+\tau\left\|\nabla \times \delta e^{2}\right\|_{\mathbf{L}^{2}}^{2}+\tau\left\|\nabla \cdot \delta e^{2}\right\|_{L^{2}}^{2}+\tau\left\|\delta e^{2}\right\|_{B}^{2}+\chi \tau\left\|\nabla \cdot e^{2}\right\|_{L^{2}}^{2}+\tau^{2}\left\|\mathcal{P}^{\frac{3}{2}}\right\|_{A}^{2} \leq \tau^{4} .
$$

By summing the above inequalities from $k=2$ to $K$ and by applying the discrete Grönwall lemma we finally obtain the following error bound:

$$
\begin{aligned}
\left\|\delta e_{\tau}\right\|_{\ell^{\infty}\left(\mathbf{L}^{2}\right)}^{2}+\tau\left\|\nabla \times \delta e_{\tau}\right\|_{\ell^{\infty}\left(\mathbf{L}^{2}\right)}^{2} & +\left\|\nabla \times \delta \bar{e}_{\tau}\right\|_{\ell^{2}\left(\mathbf{L}^{2}\right)}^{2}+\chi \tau\left\|\nabla \cdot e_{\tau}\right\|_{\ell^{\infty}\left(L^{2}\right)}^{2} \\
& +\tau^{2}\left\|\mathcal{P}_{\tau}\right\|_{\ell^{\infty}(A)}^{2} \leq c \tau^{4} .
\end{aligned}
$$

This completes the proof.

4.3. Error estimates. Having obtained the estimate of Theorem 4.1 we can now show that the rotational version of the algorithm provides a better order of convergence for the velocity in the $\ell^{2}\left(\mathbf{L}^{2}\right)$-norm, at least in two space dimensions. To this end, let us denote by $\bar{\psi}_{\tau}$ the sequence whose generic term is $\bar{\psi}^{k+\frac{1}{2}}:=\frac{1}{2}\left(\psi^{k+1}+\psi^{k}\right)$.

Theorem $4.2\left(\ell^{2}\left(\mathbf{L}^{2}\right)\right.$ Velocity Estimates). Assume that the space dimension is two. Under the assumptions of Theorem 4.1, the solution $\left(u_{\tau}, p_{\tau}\right)$ of the scheme (2.1) -(2.6) in two space dimensions satisfies

$$
\left\|\mathfrak{u}_{\tau}-u_{\tau}\right\|_{\ell^{2}\left(\mathbf{L}^{2}\right)} \leq c \tau^{\frac{3}{2}}
$$

Proof. The proof proceeds by a duality argument using the right-inverse Stokes operator $S$. By proceeding as in Section 3.5 we obtain (see (3.21)):

$$
\left\|\bar{e}_{\tau}\right\|_{\ell^{2}\left(\mathbf{L}^{2}\right)}^{2} \leq c\left(\tau^{4}+\left\|\bar{e}_{\tau}-P_{\mathbf{H}} \bar{e}_{\tau}\right\|_{\ell^{2}\left(\mathbf{L}^{2}\right)}^{2}\right) .
$$

The estimate (4.7) then immediately implies

$$
\left\|\bar{e}_{\tau}\right\|_{\ell^{2}\left(\mathbf{L}^{2}\right)}^{2} \leq c\left(\tau^{4}+\left\|\nabla \cdot \bar{e}_{\tau}\right\|_{\ell^{2}\left(\mathbf{L}^{2}\right)}^{2}\right) \leq c \tau^{3} .
$$

Now we observe that

$$
\left\|e^{k+1}\right\|_{\mathbf{L}^{2}}^{2}=\left\|\bar{e}^{k+\frac{1}{2}}+\frac{1}{2} \delta e^{k+1}\right\|_{\mathbf{L}^{2}}^{2} \leq \frac{3}{2}\left\|\bar{e}^{k+\frac{1}{2}}\right\|_{\mathbf{L}^{2}}^{2}+\frac{3}{4}\left\|\delta e^{k+1}\right\|_{\mathbf{L}^{2}}^{2},
$$

which, along with (4.7), implies

$$
\left\|e_{\tau}\right\|_{\ell^{2}\left(\mathbf{L}^{2}\right)}^{2} \leq \frac{3}{2}\left\|\bar{e}_{\tau}\right\|_{\ell^{2}\left(\mathbf{L}^{2}\right)}^{2}+c\left\|\delta e^{k+1}\right\|_{\ell^{\infty}\left(\mathbf{L}^{2}\right)}^{2} \leq c \tau^{3},
$$


which completes the argument.

Let us now show convergence of the velocity in the $\ell^{2}\left(\mathbf{H}^{1}\right)$-norm without any restriction on the space dimension.

Theorem $4.3\left(\ell^{2}\left(\mathbf{H}^{1}\right)\right.$ Velocity Estimates). Under the assumptions of Theorem 4.1, the solution $\left(u_{\tau}, p_{\tau}\right)$ of the scheme (2.1)-(2.6) in two space dimensions and (2.9)(2.15) in three space dimensions satisfies

$$
\left\|\overline{\mathfrak{u}}_{\tau}-\bar{u}_{\tau}\right\|_{\ell^{\infty}\left(\mathbf{H}^{1}\right)} \leq c \tau .
$$

Proof. Observe first that the following holds for all $k \in\{0, \ldots, K\}$ :

$$
\left\|\nabla \times \bar{e}^{k+\frac{1}{2}}\right\|_{\mathbf{L}^{2}} \leq \sum_{i=1}^{k}\left\|\nabla \times \delta \bar{e}^{i+\frac{1}{2}}\right\|_{\mathbf{L}^{2}}+\left\|\nabla \times \bar{e}^{-\frac{1}{2}}\right\|_{\mathbf{L}^{2}},
$$

which implies

$$
\left\|\nabla \times \bar{e}^{k+\frac{1}{2}}\right\|_{\mathbf{L}^{2}} \leq c \tau^{-1}\left\|\nabla \times \delta \bar{e}_{\tau}\right\|_{\ell^{2}\left(\mathbf{L}^{2}\right)}+c \tau \leq c\left(\tau^{-1} \tau^{2}+\tau\right) \leq c \tau,
$$

and, owing to (1.7), this concludes the proof since we have already established that $\left\|\nabla \cdot \bar{e}^{k+\frac{1}{2}}\right\|_{L^{2}} \leq c \tau^{\frac{3}{2}}$.

Remark 4.1 (Pressure Error Estimates). The same methods and ideas used in Section 3.4 can be invoked to show that the pressure satisfies the following estimate:

$$
\left\|\epsilon_{\tau}\right\|_{\ell^{2}(\Delta)} \leq c \tau \text {. }
$$

We omit the details for the sake of brevity.

Remark 4.2. Whether Theorem 4.2 holds in three space dimensions is not clear. The main obstacle is the splitting error induced by the splitting of the momentum equation. Based on our numerical experiments, we conjecture that both the error estimates in Theorem 4.2 and Theorem 4.3 can be improved by a $\tau^{\frac{1}{2}}$ factor irrespective of the space dimension.

\section{Other time MARCHING TECHNIQUeS}

As mentioned in Remark 2.7, the velocity update (2.10)-(2.13) is a sequence of three approximations of the momentum equation where each approximation consists of evaluating the second derivative in one of the spatial directions implicitly with the Crank-Nicolson scheme whereas in the other directions it either employs the solution from the previous time level, if no implicit approximation is yet computed in the given direction, or uses the already computed implicit approximations. This observation leads us to propose the following split version of the second-order backward difference scheme (BDF2) to approximate the momentum equation:

$$
\begin{gathered}
\frac{3 \eta^{k+1}-4 u^{k}+u^{k-1}}{2 \tau}-\partial_{x x} \eta^{k+1}-\left(\partial_{y y}+\partial_{z z}\right) u^{k}+\nabla p^{\star, k+1}=f^{k+1}, \\
\frac{3 \zeta^{k+1}-4 u^{k}+u^{k-1}}{2 \tau}-\partial_{x x} \eta^{k+1}-\partial_{y y} \zeta^{k+1}-\partial_{z z} u^{k}+\nabla p^{\star, k+1}=f^{k+1}, \\
\frac{3 u^{k+1}-4 u^{k}+u^{k-1}}{2 \tau}-\partial_{x x} \eta^{k+1}-\partial_{y y} \zeta^{k+1}-\partial_{z z} u^{k+1}+\nabla p^{\star, k+1}=f^{k+1} .
\end{gathered}
$$

We now write the full BDF2 algorithm in a form similar to (2.9) -(2.15). To simplify the presentation, let us assume that proper approximations of the velocity and the pressure time derivative are available at $t=-\tau$ and $t=0$. If these 
quantities are not available, we start the scheme with a lower-order approximation at the first time step in order to compute those approximations.

- Pressure predictor: Denoting by $\mathfrak{p}_{0}$ the pressure field at $t=0$, by $\phi^{\star, 0}$ an approximation of $\tau \partial_{t} \mathfrak{p}(0)$, and by $\phi^{\star,-1}$ an approximation of $\tau \partial_{t} \mathfrak{p}(-\tau)$ the algorithm is initialized by setting $p^{0}=\mathfrak{p}_{0}, \phi^{0}=\phi^{\star, 0}$, and $\phi^{-1}=\phi^{\star,-1}$. Then for all $k \geq 0$ a pressure predictor is computed as follows:

$$
p^{\star, k+1}=p^{k}+\frac{4}{3} \phi^{k}-\frac{1}{3} \phi^{k-1} .
$$

- Velocity update: The velocity update is computed by solving the following series of one-dimensional problems: Find $\xi^{k+1}, \eta^{k+1}, \zeta^{k+1}$, and $u^{k+1}$ such that

$$
\begin{aligned}
& \frac{3 \xi^{k+1}-4 u^{k}+u^{k-1}}{2 \tau}-\Delta u^{k}+\nabla p^{\star, k+1}=f^{k+1},\left.\quad \xi^{k+1}\right|_{\partial \Omega}=0, \\
& \frac{3\left(\eta^{k+1}-\xi^{k+1}\right)}{2 \tau}-\partial_{x x}\left(\eta^{k+1}-u^{k}\right)=0,\left.\quad \eta^{k+1}\right|_{x=0,1}=0, \\
& \\
& \frac{3\left(\zeta^{k+1}-\eta^{k+1}\right)}{2 \tau}-\partial_{y y}\left(\zeta^{k+1}-u^{k}\right)=0,\left.\quad \zeta^{k+1}\right|_{y=0,1}=0, \\
& \frac{3\left(u^{k+1}-\zeta^{k+1}\right)}{2 \tau}-\partial_{z z}\left(u^{k+1}-u^{k}\right)=0,\left.\quad u^{k+1}\right|_{z=0,1}=0 .
\end{aligned}
$$

- Penalty step: The pressure-correction $\phi^{k+1}$ is computed by solving

$$
A \phi^{k+1}=-\frac{3}{2 \tau} \nabla \cdot u^{k+1} .
$$

- Pressure update: The pressure is updated as follows:

$$
p^{k+1}=p^{k}+\phi^{k+1}-\chi \nabla \cdot \bar{u}^{k+1} .
$$

Note that this scheme is formally second-order consistent because eliminating the intermediate velocities results in a second-order perturbation of the classical pressurecorrection BDF2 scheme. Numerical experiments show that this algorithm is indeed unconditionally stable when tested on the unsteady Stokes problem and its rate of convergence is similar to that of (2.9)-(2.15).

\section{NumericAl EXPERIMENTS}

We report in this section numerical tests aiming at evaluating the performance of the algorithms (2.1)-(2.6) with $A=\left(1-\partial_{x x}\right)\left(1-\partial_{y y}\right)$ in two space dimensions and (2.9) - (2.15) with $A=\left(1-\partial_{x x}\right)\left(1-\partial_{y y}\right)\left(1-\partial_{z z}\right)$ in three space dimensions. The space approximation is done using the MAC stencil.

6.1. Accuracy tests. The standard and the rotational versions of the scheme (2.1) - (2.6) have been tested numerically on a two-dimensional analytic solution and the results have been reported in [11]. The rate of convergence with respect to $\tau$ for the velocity in the $\mathbf{L}^{2}$-norm for both versions of the method is about 1.8 or higher, whereas for the pressure in the $L^{2}$-norm it is about 1.85 for the rotational version and about 1.5 for the standard version.

We now investigate the convergence rates in three space dimensions in $\Omega=(0,1)^{3}$ using the following solution of the unsteady Stokes problem (with the appropriate 
source term):

$$
\begin{aligned}
& \mathfrak{u}_{1}=(\sin x \cos y \sin z-\sin x \sin y \cos z) \sin t \\
& \mathfrak{u}_{2}=(\sin x \sin y \cos z-\cos x \sin y \sin z) \sin t, \\
& \mathfrak{u}_{3}=(\cos x \sin y \sin z-\sin x \cos y \sin z) \sin t, \\
& \mathfrak{p}=\cos (x+y+z+t)
\end{aligned}
$$
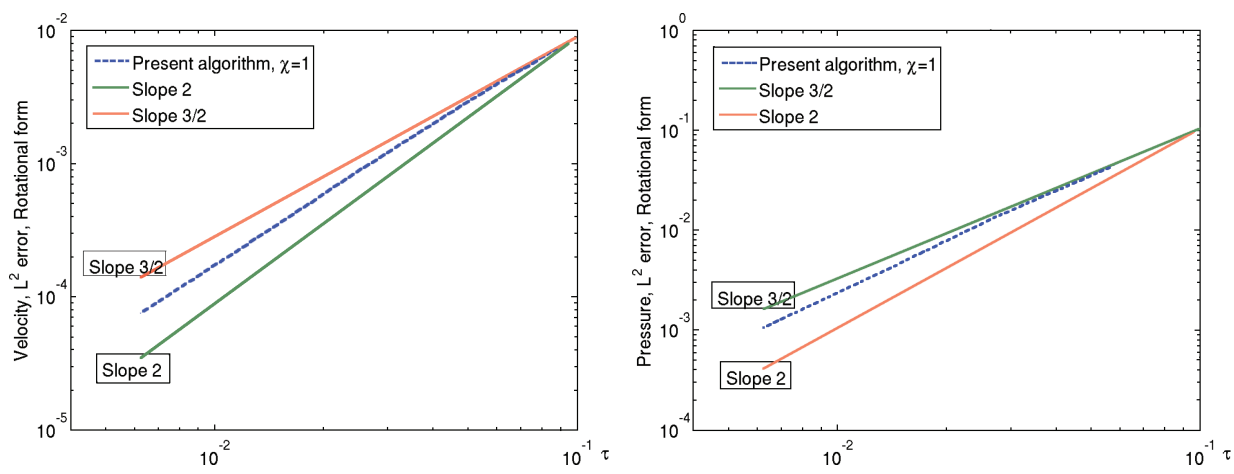

Figure 1. Rotational form $(\chi=1)$. Left: $\mathbf{L}^{2}$-norm of the error on the velocity (dashed line) at $T=2$ on a uniform grid, $100 \times 100 \times 100$; Right: $L^{2}$-norm of the error on the pressure (dashed line).

We display in the left panel of Figure 1 the $\mathbf{L}^{2}$-norm of the error on the velocity at $T=2$ versus the time step $\tau$ for the rotational scheme with $\chi=1$. The $L^{2}$ norm of the error on the pressure is displayed in the right panel of the figure. The convergence rate on the velocity varies between 1.6 and 1.8 while the convergence rate on the pressure is comprised between 1.5 and 1.7. From tests not reported here, we have observed that the standard version of the scheme has a convergence rate between 1.6 and 1.7 for the velocity and a convergence rate between 1.25 and 1.4 for the pressure. These results suggest that the actual convergence rates of both schemes are higher than those theoretically estimated above. However, at the present it is unclear how to improve these estimates.

6.2. Splitting vs. projection. To further illustrate the convergence properties of the present schemes we now compare it with its unsplit pressure-correction counterpart, i.e., the momentum equation is unsplit and the pressure correction is computed by solving the Poisson problem $\left(A=-\Delta_{N}\right)$. The comparison is done in two space dimensions in $\Omega=(0,1)^{2}$ on the following analytical solution:

$$
\mathfrak{u}=(\sin x \sin (y+t), \cos x \cos (y+t)), \quad \mathfrak{p}=\cos x \sin (y+t) .
$$

We show in Figure 2 the error on the velocity and the pressure as functions of $\tau$ for the unsplit second-order projection and the corresponding results using the present direction splitting schemes. Clearly, both the standard and the rotational versions of the direction splitting schemes produce results that are very similar to those produced by their unsplit counterpart. The largest differences are observed on the velocity for the standard version of the schemes. But, even in this case, the direction splitting produces errors which are only between 1.2 and 2 times larger 

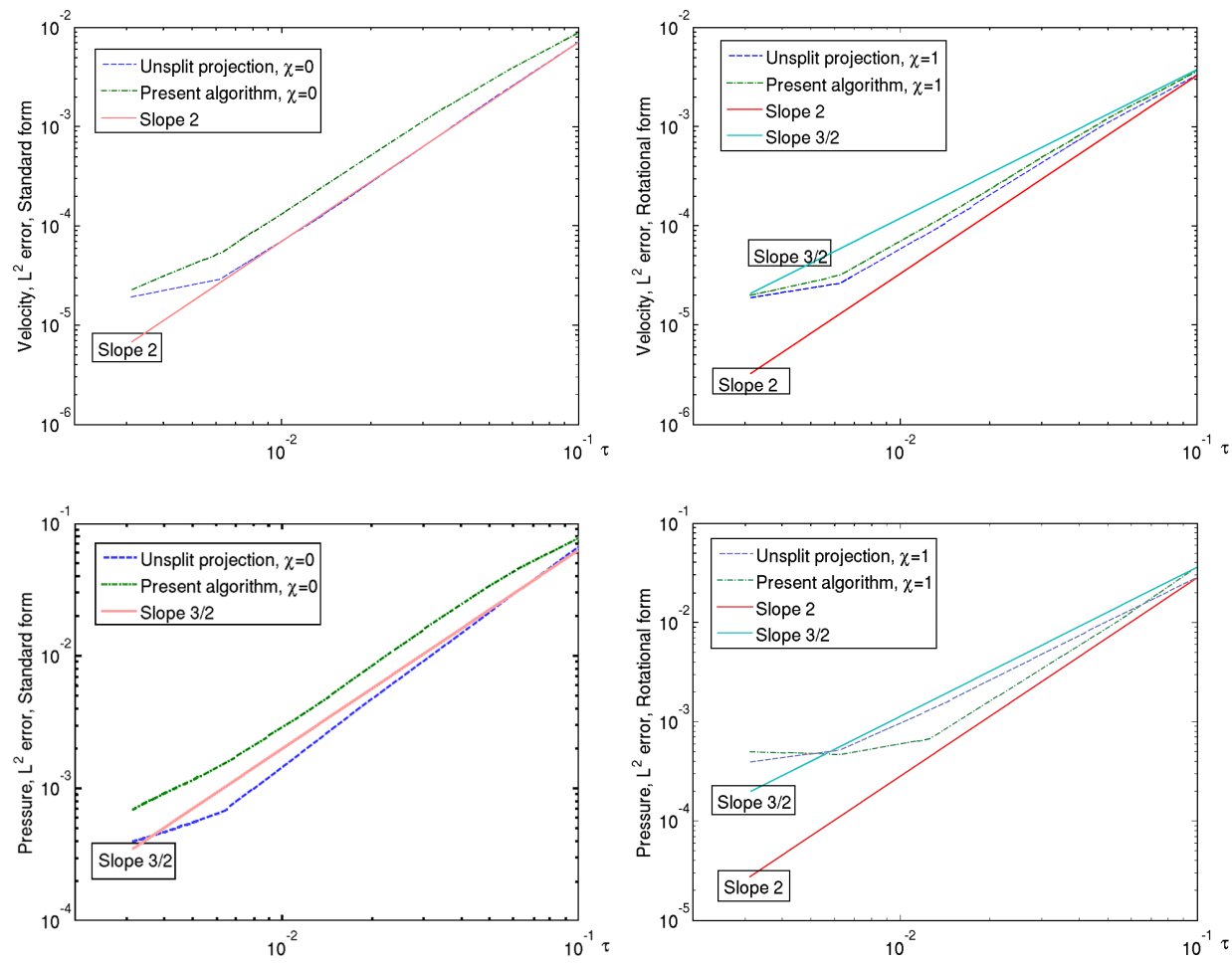

FiguRe 2. $\mathbf{L}^{2}$-norm of the error on the velocity (top) and pressure (bottom) at $T=2$ on a uniform $40 \times 40$ grid; Left: unsplit projection scheme in standard form (dashed line) and scheme (2.7)-(2.6) with $\chi=0$ (dash-dotted line). Right: unsplit projection scheme in rotational form with $\chi=1$ (dashed line) and scheme (2.7)-(2.6) with $\chi=1$ (dash-dotted line).

than the errors produced by the classical standard scheme. The computational complexity of the present schemes, however, is significantly lower.

6.3. Lid driven cavity. We compare in this section the performance of the direction splitting algorithm with its unsplit pressure-correction counterpart on the so-called lid driven cavity. The computational domain is $\Omega=(0,1)^{2}$. The boundary conditions are $\left.u\right|_{x=0,1, y=0}=0,\left.u\right|_{y=1}=1$ and $\left.v\right|_{\partial \Omega}=0$. The computation is done at Reynolds number $R_{e}=100$ on a MAC grid composed of $40 \times 40$ nodes and with time step $\tau=0.01$. The advection term is computed by means of the explicit second-order Adams-Bashforth approximation; the actual direction splitting algorithm used for these tests is (2.1)-(2.16)-(2.3)-(2.4)-(2.5)-(2.6). The comparison between the two codes is done at $t=1$ and $t=10$.

We show in Figure 3 the horizontal and vertical profiles of the velocity alongside the vertical/horizontal lines through the center of the cavity. The results of the two schemes (unsplit and split) are very close to each other; detailed examination (not reported here) shows that the two sets of results differ in the fourth decimal digit. For comparison, we also display with o symbols the result of the split scheme on a 
MAC grid composed of $200 \times 200$ nodes and with time step $\tau=0.0025$. The three sets of results are visually indistinguishable.
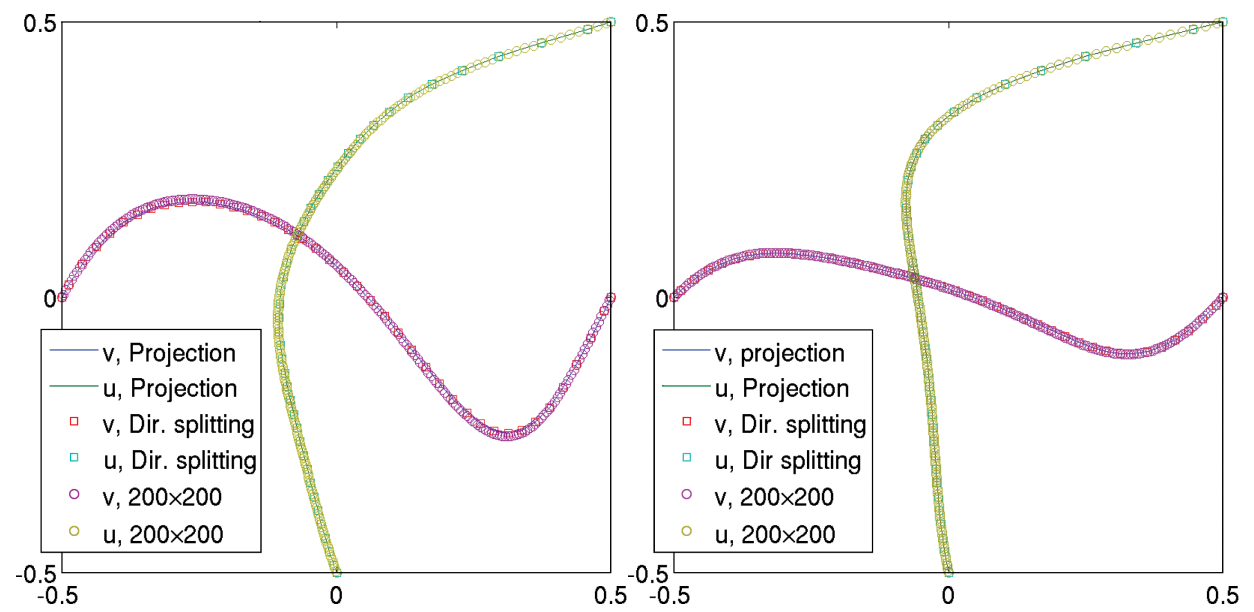

FiguRE 3. The horizontal (resp. vertical) profiles of the velocity along the vertical (resp. horizontal) lines through the center of the cavity at $R_{e}=100$ and at $t=1$ (right panel) and $t=10$ (left panel) on a $40 \times 40 \mathrm{MAC}$ grid, $\tau=0.01$. Unsplit projection scheme (solid line); direction splitting scheme ( $\square, \square$ symbols); direction splitting on a $200 \times 200$ MAC grid with $\tau=0.0025$ (॰, ○ symbols).

6.4. Backward facing step. Finally, the new direction splitting method is validated on the two-dimensional flow over a backward-facing step. Extensive experimental and computational data on this flow is available in [1] and [19. Here we compute the solution to this problem in a rectangular cavity of size $1 \times 16$ with a uniform grid of size $h=0.005$ and a time step $\tau=0.001$. We prescribe the fully developed parabolic profile with maximum velocity $\frac{3}{2}$ at the upper half of the inflow side and we prescribe the no-slip condition at the lower half. At the outlet we impose zero-Neumann conditions on the velocity and the zero Dirichlet condition on the pressure. One important characteristic of the flow is the length of the recirculation zone behind the step, say $r$. We report in Table 1 the results of the present computations at Reynolds numbers (based on the channel height) $R_{e}=100,200$ and 400 and we compare these results with those from [19]. The present scheme yields results which are in a very good agreement with the existing data.

6.5. Parallel implementation. We have implemented a parallel version of the algorithm (2.9) -(2.15), with $A=\left(1-\partial_{x x}\right)\left(1-\partial_{y y}\right)\left(1-\partial_{z z}\right)$, using the MAC stencil together with central differences for the first- and second-order derivatives. The algorithm has been implemented in parallel on a Cartesian domain decomposition using MPI. Allthe one-dimensional linear systems are solved in parallel by means of direct solves of the Schur complement induced by the domain decomposition. We have verified that the weak scalability of the code is quasi-perfect up to the maximum number of processors that were available to us without special request for allocation, i.e., 1024 processors. The reader is referred to [16] for more details on scalability tests. 
TABLE 1. Flow over a backward-facing step. Re-attachment length $r$ divided by the step height $s$ as a function of the Reynolds number $R_{e}$ for the present computations and for the computations of Kim and Moin [19].

\begin{tabular}{||c|c|c||}
\hline$R_{e}$ & \multicolumn{2}{|c||}{$r / s$} \\
\hline \multirow{2}{*}{100} & Current result & Result in [19] \\
\cline { 2 - 3 } 200 & 3.22 & 3.2 \\
\hline 200 & 5.33 & 5.3 \\
\hline 400 & 8.6 & 8.6 \\
\hline
\end{tabular}

Extensive numerical tests have shown that the algorithm is stable under CFL condition in the Navier-Stokes regime. We have computed a highly accurate benchmark solution for the start-up flow in a three-dimensional impulsively started liddriven cavity of aspect ratio $1 \times 1 \times 2$ at Reynolds numbers 1000 and 5000 . Successive refinements have shown that the velocity field is four digit accurate at $R_{e}=5000$ for dimensionless times $t=4,8$ and 12. The computations have been done in parallel on 512 processors on adapted grids of up to 2 billion nodes in three space dimensions. All of these numerical experiments are reported in [17].

6.6. Further developments. We believe that the algorithm presented in the present paper has a lot of potential for further developments; we are thinking in particular of academic problems that can be solved in simple geometries with regular grids, e.g., simulation of turbulent flows in the atmosphere and in the ocean, simulation of multiphase flows, stratified flows, variable density flows, combustion, solution of subgrid problems as part of an homogenization procedure, etc.

As described in the present paper, the algorithm is suitable only for domains of simple shape. However, there are possibilities to impose boundary conditions either via penalty methods, or fictitious domain techniques, or via directional adjustment of the grid at the boundary. The authors have implemented the directional adjustment procedure and have observed that the resulting scheme is unconditionally stable and convergent for the time-dependent Stokes problem. These results will be reported elsewhere.

\section{REFERENCES}

1. B.F. Armally, F. Durst, J.C.F. Pereira, and B. Schönung, Experimental and theoretical investigation of backward-facing step flow, J. Fluid Mech. 127 (1983), 473-496.

2. J.H. Bramble, J.E. Pasciak, and J. Xu, Parallel multilevel preconditioners., Math. Comp. 55 (1990), no. 191, 1-22 (English). MR1023042 (90k:65170)

3. J.H. Bramble and X. Zhang, The analysis of multigrid methods, Handbook of numerical analysis, Vol. VII, Handbook Numer. Anal., VII, North-Holland, Amsterdam, 2000, pp. 173415. MR $1804746(2001 \mathrm{~m}: 65183)$

4. A.J. Chorin, Numerical solution of the Navier-Stokes equations, Math. Comp. 22 (1968), 745-762. MR0242392 (39:3723)

5. M. Dauge, Stationary Stokes and Navier-Stokes systems on two- or three-dimensional domains with corners. I. Linearized equations, SIAM J. Math. Anal. 20 (1989), no. 1, 74-97. MR977489 (90b:35191)

6. J. Douglas, Jr., Alternating direction methods for three space variables, Numer. Math. 4 (1962), 41-63. MR0136083 (24:B2122)

7. A. Ern and J.-L. Guermond, Theory and practice of finite elements, Applied Mathematical Sciences, vol. 159, Springer-Verlag, New York, 2004. MR2050138 
8. V. Girault and P.-A. Raviart, Finite element methods for Navier-Stokes equations. theory and algorithms, Springer Series in Computational Mathematics, Springer-Verlag, Berlin, Germany, 1986. MR88b:65129

9. J.-L. Guermond, Un résultat de convergence d'ordre deux en temps pour l'approximation des équations de Navier-Stokes par une technique de projection incrémentale, M2AN Math. Model. Numer. Anal. 33 (1999), no. 1, 169-189, Also in C. R. Acad. Sci. Paris, Série I, 325:1329-1332, 1997. MR:2000k:65171

10. J.-L. Guermond, P. Minev, and J. Shen, An overview of projection methods for incompressible flows, Comput. Methods Appl. Mech. Engrg. 195 (2006), no. 44-47, 6011-6045. MR2250931 (2007g:76157)

11. J.-L. Guermond and P.D. Minev, A new class of fractional step techniques for the incompressible Navier-Stokes equations using direction splitting, Comptes Rendus Mathematique 348 (2010), no. 9-10, 581-585. MR2645177 (2011f:76128)

12. J.-L. Guermond and L. Quartapelle, Calculation of incompressible viscous flows by an unconditionally stable projection FEM, J. Comput. Phys. 132 (1997), no. 1, 12-33. MR.1440332 (97k:76066)

13. J.-L. Guermond and A. Salgado, A splitting method for incompressible flows with variable density based on a pressure Poisson equation, Journal of Computational Physics 228 (2009), no. 8, 2834-2846. MR2509298 (2010m:65174)

14. J.-L. Guermond and A.J. Salgado, Error analysis of a fractional time-stepping technique for incompressible flows with variable density, SIAM Journal on Numerical Analysis 49 (2011), no. 3, 917-944. MR 2802553

15. J.-L. Guermond and J. Shen, On the error estimates for the rotational pressure-correction projection methods, Math. Comp. 73 (2004), no. 248, 1719-1737 (electronic). MR2059733

16. J.L. Guermond and P.D. Minev, A new class of massively parallel direction splitting for the incompressible Navier-Stokes equations, Computer Methods in Applied Mechanics and Engineering 200 (2011), no. 23-24, 2083-2093. MR2795163

17. Start-up flow in a three-dimensional lid-driven cavity by means of a massively parallel direction splitting algorithm, Int. J. Numer. Meth. Fluids (2011), Available online at http://onlinelibrary.wiley.com/doi/10.1002/fld.2583.

18. J.G. Heywood and R. Rannacher, Finite element approximation of the nonstationary NavierStokes problem. I. Regularity of solutions and second-order error estimates for spatial discretization, SIAM J. Numer. Anal. 19 (1982), no. 2, 275-311. MR650052 (83d:65260)

19. J. Kim and P. Moin, Application of a fractional-step method to incompressible Navier-Stokes equations, J. Comput. Phys. 59 (1985), no. 2, 308-323. MR0796611(87a:76046)

20. T. Lu, P. Neittaanmäki, and X.-C. Tai, A parallel splitting up method and its application to Navier-Stokes equations, Appl. Math. Lett. 4 (1991), no. 2, 25-29. MR1095644

21. __ A parallel splitting-up method for partial differential equations and its applications to Navier-Stokes equations, RAIRO Modél. Math. Anal. Numér. 26 (1992), no. 6, 673-708. MR.1183413 (93i:65111)

22. D. W. Peaceman and H.H. Rachford, Jr., The numerical solution of parabolic and elliptic differential equations, J. Soc. Indust. Appl. Math. 3 (1955), 28-41. MR0071874 (17,196d)

23. A. Prohl, Projection and quasi-compressibility methods for solving the incompressible NavierStokes equations, Advances in Numerical Mathematics, B. G. Teubner, Stuttgart, 1997. MR.1472237(98k:65058)

24. R. Rannacher, On Chorin's projection method for the incompressible Navier-Stokes equations, The Navier-Stokes Equations II-Theory and Numerical Methods (Oberwolfach, 1991), Lecture Notes in Math., vol. 1530, Springer, Berlin, Germany, 1992, pp. 167-183. MR95a:65149

25. J. Shen, On error estimates of some higher order projection and penalty-projection methods for Navier-Stokes equations, Numer. Math. 62 (1992), no. 1, 49-73. MR.1159045 (93a:35122)

26. - On error estimates of projection methods for the Navier-Stokes equations: secondorder schemes, Math. Comp. 65 (1996), no. 215, 1039-1065. MR1348047 (96j:65091)

27. R. Temam, Sur l'approximation de la solution des équations de Navier-Stokes par la méthode des pas fractionnaires ii, Arch. Rat. Mech. Anal. 33 (1969), 377-385. MR0244654 (39:5968)

28. R. Temam, Navier-Stokes equations, AMS Chelsea Publishing, Providence, RI, 2001, Theory and numerical analysis, Reprint of the 1984 edition. MR 1846644 (2002j:76001) 
29. L.J.P. Timmermans, P.D. Minev, and F.N. van de Vosse, An approximate projection scheme for incompressible flow using spectral elements, Int. J. Numer. Methods Fluids 22 (1996), 673-688.

30. N.N. Yanenko, B.G. Kuznetsov, and Sh. Smagulov, On the approximation of the Navier-Stokes equations for an incompressible fluid by evolutionary-type equations, Numerical methods in fluid dynamics, "Mir", Moscow, 1984, pp. 290-314. MR804995 (87a:65156)

Department of Mathematics, Texas A\&M University, 3368 TAMU, College Station, TeXas 77843-3368. On LEAVE From CNRS, France

E-mail address: guermond@math.tamu.edu

Department of Mathematical and Statistical Sciences, University of Alberta, Edmonton, Alberta Canada T6G 2G1

E-mail address: minev@ualberta.ca

Department of Mathematics, University of Maryland, College Park, Maryland 20742

E-mail address: abnersg@math.umd.edu 